\title{
Universal Majorana thermoelectric noise
}

\author{
Sergey Smirnov \\ P. N. Lebedev Physical Institute of the Russian Academy of Sciences, 119991 Moscow, Russid*
}

(Dated: July 8, 2021)

\begin{abstract}
Thermoelectric phenomena resulting from an interplay between particle flows induced by electric fields and temperature inhomogeneities are extremely insightful as a tool providing substantial knowledge about the microscopic structure of a given system. Tuning, e.g., parameters of a nanoscopic system coupled via tunneling mechanisms to two contacts one may achieve various situations where the electric current induced by an external bias voltage competes with the electric current excited by the temperature difference of the two contacts. Even more exciting physics emerges when the system's electronic degrees freedom split to form Majorana fermions which make the thermoelectric dynamics universal. Here we propose revealing this unique universal signatures of Majorana fermions in strongly nonequilibrium quantum dots via noise of the thermoelectric transport beyond linear response. It is demonstrated that whereas mean thermoelectric quantities are only universal at large bias voltages, the noise of the electric current excited by an external bias voltage and the temperature difference of the contacts is universal at any bias voltage. We provide truly universal, i.e. independent of the system's parameters, thermoelectric ratios between nonlinear response coefficients of the noise and mean current at large bias voltages where experiments may easily be performed to uniquely detect these truly universal Majorana thermoelectric signatures.
\end{abstract}

\section{INTRODUCTION}

Originally proposed [1] in the late 1930s the Majorana representation [2], making the Dirac equation real, has since then always been a challenge for experiments on elementary particles to reveal, e.g. via neutrinoless double beta decay, a fundamental particle representing on equal footing its own antiparticle. Referred to as Majorana fermions, these are hypothetical spin-1/2 neutral fundamental particles with neutrinos as possible candidates for massive Majorana particles.

In parallel to Majorana challenges within the particle physics another way to implement Majorana fermions is to construct condensed matter systems with quasiparticles being identical to their own antiquasiparticles. This is indeed possible when a system acquires a finite superconducting order parameter. Here Dirac fermions may become highly nonlocal in real space, i.e. they may split, or fractionalize, into two Majorana fermions localized at the system's edges. Although these zero energy Majorana bound states are not fundamental particles as in the case of the particle physics, they are effectively quasiparticles which are their own antiquasiparticles. Despite their non-abelian statistics these zero energy Majorana bound states are still referred to as Majorana fermions. The Kitaev model [3] is an example of a one-dimensional condensed matter system hosting two Majorana fermions at its ends. The topological superconducting phase realized in the Kitaev model has various [4] 6 , physical implementations among which systems based on topological insulators [7, 8, and spin-orbit coupled semiconductors 9, 10, are of particular interest.

Once implemented in a condensed matter setup Majorana fermions need an experimental proof of their exis-

\footnotetext{
* sergej.physik@gmail.com, ssmirnov@sci.lebedev.ru
}

tence via unique signatures characteristic of exclusively these fractionalized quasiparticles. To this end transport experiments offer a relatively simple way to detect unique Majorana signatures in a given condensed matter system.

The majority of transport proposals focus on mean electric current often providing low-energy behavior of the system's electrical conductance. Examples are given by superconductor-Luttinger liquid junctions [11, Kondo effect in quantum dots side coupled to a topological superconductor supporting Majorana fermions at its ends [12], driven topological superconductors [13], Josephson junctions on surfaces of three-dimensional topological insulators 14, Kondo effect in topological superconductorquantum dot-normal lead junction [15, normal metalsuperconducting semiconductor-normal metal structures [16], spinon-antispinon systems [17], Coulomb blockaded systems [18], disordered Josephson junctions in tilted magnetic fields [19] and many others.

Transport experiments [20, 21] oriented on measurements of the mean current, in particular, on its lowenergy behavior are steadily improving their analysis of the zero bias anomaly present in the electrical conductance from which stronger signatures of Majoranas may be extracted 22. Although it is often necessary to perform additional analysis to disentangle the Majorana physics from possible interaction induced effects, such as the Kondo effect, or from effects related to partiallyseparated Andreev bound states 23, 24, recent works 25, 26] suggest an alternative proof of the nonlocal nature and topological protection of Majorana zero modes via local measurements of the zero bias conductance.

To avoid uncertainty and make transport experiments provide more unique signatures of Majorana fermions one might, in conjunction with measurements of zero bias conductance, resort to measurements of the electric current fluctuations. Here there are not as many proposals 27 30] for Majorana noise as for the mean electric cur- 
rent. Again the most attention is paid to the low-energy behavior of the electric current noise. Because of the fluctuation-dissipation theorem [31 33 this equilibrium noise, accessed within the system's linear response, is not independent of the mean electric current. Beyond linear response the electric current fluctuates independently of its mean value and thus this nonequilibrium noise is able to provide alternative Majorana fingerprints which, what is remarkable, turn out to be of universal nature 34, 35. In particular, in Ref. 35. it has been demonstrated that this universal Majorana noise is characterized by two effective charges $e_{l}^{*}=e / 2$ and $e_{h}^{*}=3 e / 2$ at low and high energies, respectively. It has also been shown that the low-energy Majorana effective charge $e_{l}^{*}=e / 2$, whose Majorana nature is additionally confirmed via Majorana tunneling entropy calculations [36], might be sensitive to thermal fluctuations while the high-energy Majorana effective charge $e_{h}^{*}=3 e / 2$ is accessed at high bias voltages which protect this effective charge from thermal noise and, therefore, its measurement may be performed at relatively high temperatures easily reachable in modern laboratories. It is important to note that modern experiments have already reached such a high level at which one can get the effective charge with very high accuracy as for example in experiments on quantum dots where the effective charge has been measured in the Kondo regime 37 .

Another realm of transport experiments is provided by systems hosting Majorana fermions and possessing temperature inhomogeneities. Here examples include heat conduction in a Majorana metal, where the heat conduction happens via Majorana fermions bound to defects 38, and thermal quantum Hall effect 39, where a net heat current results from a temperature difference between the edges which support Majorana chiral edge modes arising for example in a chiral $p$-wave superconductor. The latter case of dispersive Majorana fermions is particularly attractive for future research on thermoelectric phenomena in systems where Majorana dispersion relations may undergo qualitative changes such as, for example, the evolution of a Majorana conic dispersion relation into a Majorana arc shaped dispersion relation 40. In the context of nanoscopic systems one is usually interested in setups where a nanoscopic system interacts via tunneling mechanisms with two normal metals playing the role of the contacts. An external bias voltage is applied to these contacts which may also have different temperatures. Here one may easily tune the system's parameters making the electric current induced by the bias voltage and the electric current excited by the temperature difference interfere with one another leading to a highly nontrivial thermoelectric transport [41, 42]. When a nanoscopic setup is designed to host Majorana degrees of freedom, the system's thermoelectric response undergoes essential changes which might be used to identify Majorana fermions in, e.g., normal metal-quantum dot-Majorana bound states junction [43, Majorana-sidecoupled quantum dots [4, 45, Majorana bound states system coupled to two normal leads [46].

Up to now Majorana thermoelectric transport has been investigated via analysis of the mean electric current often restricted to its low-energy behavior to obtain linear conductances. Fluctuations of the electric current excited by both the bias voltage and temperature difference have not been addressed. At the same time, as discussed above, fluctuations provide much more unique fingerprints of a given system. Therefore, thermoelectric noise is an important and highly conclusive tool to uniquely reveal whether Majorana fermions are present in a given nanoscopic system.

In this work we explore fluctuations of thermoelectric transport through quantum dots interacting via tunneling mechanisms with two contacts and one end of a onedimensional Kitaev's chain, supporting two Majorana fermions at its ends. The contacts are normal metals to which an external bias voltage is applied and in general the temperatures of the two contacts are not identical. We obtain for this system both the mean electric current and thermoelectric noise as well as their nonlinear response coefficients in the transport regime governed by Majorana degrees of freedom. We find 1) that mean thermoelectric quantities become universal only for large bias voltages while having non-universal behavior below the energy scale characterizing the overlap of the Majorana modes; 2) that in contrast to the mean quantities thermoelectric noise is universal in the whole voltage range when the dynamics is essentially governed by Majorana bound states; 3 ) that the differential thermoelectric noise has a universal two plateau structure with the truly universal values of the plateaus $\left(e^{3} / h\right)[1+\ln (2)]$ and $\left(e^{3} / h\right)\left[1+\ln \left(2^{1 / 2}\right)\right]$ depending on whether the ratio between the bias voltage and the thermal voltage is less than or greater than one; 4) analytical universal high-energy asymptotics of the nonlinear response coefficients of the thermoelectric noise; 5) universal ratios between nonlinear response coefficients of the thermoelectric noise and mean current; 6) that at large bias voltages these thermoelectric ratios saturate to truly universal constants independent of the bias voltage; 7) analytical values of these constants; 8) that these values are protected by high bias voltages, making them robust against thermal noise and, therefore, these values represent unique truly universal Majorana thermoelectric signatures which could be measured in modern experiments even at relatively high temperatures.

The paper is organized as follows. In Section II we describe in detail an example of a system where thermoelectric transport in presence of Majorana fermions may be analyzed on the level of both mean quantities and fluctuations. The description of the system is given in terms of the Keldysh field integral most convenient to explore different correlation functions in stationary nonequilibrium which is the case here. In Section III thermoelectric transport is analyzed in terms of nonlinear response coefficients: first on the level of mean quantities, in Subsection III A and afterwards on the level of fluctuations, 


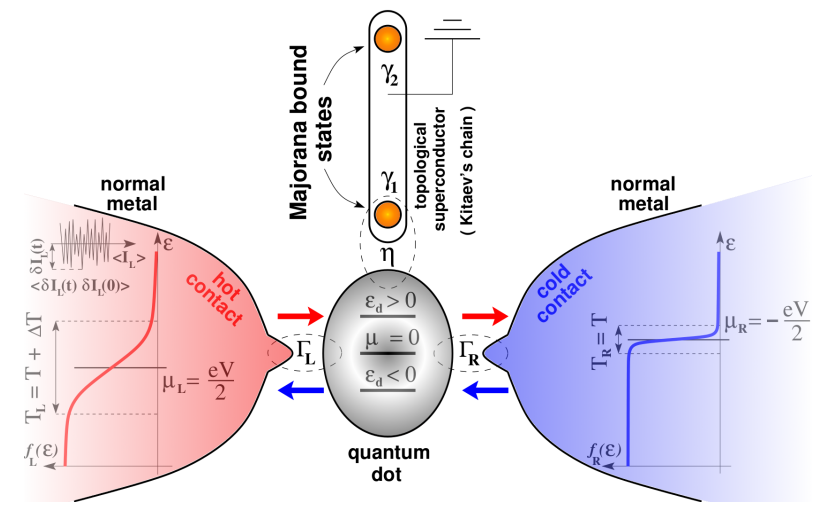

FIG. 1. The physical system represents a quantum dot whose single-particle energy level $\epsilon_{d}$ may be tuned by an external gate voltage to make the quantum dot filled $\left(\epsilon_{d}<0\right)$ or empty $\left(\epsilon_{d}>0\right)$. The left $(L)$ and right $(R)$ contacts are normal metals assumed to be in equilibrium characterized by the Fermi-Dirac distributions $f_{L, R}(\varepsilon)$ specified by the chemical potentials $\mu_{L, R}$ and the temperatures $T_{L, R}$. They are coupled via tunneling interactions (with the strengths $\Gamma_{L, R}$ ) to the quantum dot. An external bias voltage $V$ is applied to the contacts, $\mu_{L}-\mu_{R}=e V<0$. The left contact is hot (shown as red) while the right one is cold (shown as blue), $T_{L}=T+\Delta T, T_{R}=T, \Delta T \geqslant 0$. The grounded topological superconductor, implemented, e.g, by means of the Kitaev's chain, supports two Majorana modes $\gamma_{1,2}$ at its ends. One of these ends, namely the one supporting the Majorana mode $\gamma_{1}$, is coupled via another tunneling interaction (with the strength $\eta$ ) to the quantum dot. The blue and red arrows illustrate schematically the electric current flows excited by the external bias voltage $V$ and the temperature difference $\Delta T$, respectively. The noise of the electric current $S^{>}(t)$ and the mean electric current $I$ are measured in the hot (left) contact, $S^{>}(t)=\left\langle\delta I_{L}(t) \delta I_{L}(0)\right\rangle, I=\left\langle I_{L}\right\rangle$.

in Subsection IIIB. In Section IV we summarize the main results of the paper, draw conclusions and discuss some open issues. Finally, the appendix provides the main technical steps in the calculation of the current-current correlator.

\section{NONEQUILIBRIUM FIELD-THEORETIC FRAMEWORK: KELDYSH ACTION}

To explore Majorana thermoelectric transport in a nanoscopic setup we focus on a particular example of a quantum dot system shown in Fig. 1. It includes a noninteracting quantum dot with a single-particle energy level $\epsilon_{d}$ which is spin nondegenerate. Physical realization may represent a quantum dot subjected to a Zeeman field leading to a spin polarized energy level $\epsilon_{d}$. Additionally, the position of this energy level with respect to the chemical potential may be controlled by an external gate voltage so that the quantum dot is filled when $\epsilon_{d}<0$ and empty when $\epsilon_{d}>0$. The Hamiltonian of the isolated quantum dot is

$$
\hat{H}_{d}=\epsilon_{d} d^{\dagger} d,
$$

where $d$ and $d^{\dagger}$ are, respectively, the quantum dot Dirac fermion annihilation and creation operators with the usual anticommutation relations, $\{d, d\}=0,\left\{d, d^{\dagger}\right\}=1$. If, as mentioned above, a Zeeman field is applied to the quantum dot, it will destroy the correlation effects due to the Kondo effect [47] so that the latter is not important for the Majorana physics discussed below. Moreover, as is well known, the Kondo effect in the present setup would require the quantum dot to be filled $\left(\epsilon_{d}<0\right)$. However, for the case of the empty quantum $\operatorname{dot}\left(\epsilon_{d}>0\right)$ the Kondo effect does not arise. On the other side, as will be shown below (see also Ref. [35] for pure electric Majorana noise), the thermoelectric Majorana noise is universal and it does not depend on whether $\epsilon_{d}<0$ or $\epsilon_{d}>0$. Therefore, in order to completely exclude the Kondo effect from the physical setup, we will below always consider the case $\epsilon_{d}>0$.

The setup also contains two contacts labeled as left $(L)$ and right $(R)$. They represent noninteracting normal metals which are assumed to be in equilibrium characterized by the Fermi-Dirac distributions:

$$
f_{L, R}(\epsilon)=\frac{1}{\exp \left(\frac{\epsilon-\mu_{L, R}}{T_{L, R}}\right)+1},
$$

where $\mu_{L, R}$ and $T_{L, R}$ are, respectively, the chemical potentials and temperatures of the contacts. An external bias voltage $V$ may be applied to the contacts so that $\mu_{L}-\mu_{R}=e V$, where $e$ is the electron charge, and we will assume the symmetric bias, $\mu_{L, R}= \pm \mathrm{eV} / 2$, with $\mathrm{eV}<0$. The temperatures of the contacts are $T_{L}=T+\Delta T$, $T_{R}=T$ with $T \geqslant 0, \Delta T \geqslant 0$, i.e. the left contact is hot and the right contact is cold. We consider a typical setup where the two contacts are characterized by the same set of quantum numbers $k$ and energy spectrum $\epsilon_{k}$. In this case the Hamiltonian of the contacts is

$$
\hat{H}_{c}=\sum_{l=\{L, R\}} \sum_{k} \epsilon_{k} c_{l k}^{\dagger} c_{l k}
$$

where $c_{l k}$ and $c_{l k}^{\dagger}$ are, respectively, the contacts Dirac fermion annihilation and creation operators with the usual anticommutation relations, $\left\{c_{l k}, c_{l^{\prime} k^{\prime}}\right\}=0$, $\left\{c_{l k}, c_{l^{\prime} k^{\prime}}^{\dagger}\right\}=\delta_{l l^{\prime}} \delta_{k k^{\prime}}$. If the quantum dot energy level $\epsilon_{d}$ is spin polarized, i.e. it has a definite spin $\sigma$ realized via a Zeeman splitting, as mentioned above, then Eq. (3) describes the electrons in the normal metals with the same spin $\sigma$. Additionally, we assume massive contacts so that their energy spectrum is continuous and their density of states per spin may be characterized by a constant $\nu_{c} / 2$ within the energy range relevant for transport.

The quantum dot interacts with the contacts via tunneling,

$$
\hat{H}_{d-c}=\sum_{l=\{L, R\}} \sum_{k} T_{l k} c_{l k}^{\dagger} d+\text { H.c. }
$$


where the dependence of the tunneling matrix elements on the contacts quantum numbers is usually neglected, $T_{l k}=T_{l}$. The strength of the tunneling between the quantum dot and the left or right contact is characterized by the quantity $\Gamma_{l} \equiv \pi \nu_{c}\left|T_{l}\right|^{2}$ while the total tunneling strength is defined as $\Gamma \equiv \sum_{l=\{L, R\}} \Gamma_{l}=\Gamma_{L}+\Gamma_{R}$.

The final constituent of the setup is a grounded topological superconductor implementing the Kitaev's onedimensional chain supporting two Majorana zero modes localized at its edges. Due to a finite length of the topological superconductor these Majorana bound states may have a finite overlap with a characteristic energy scale $\xi$. For large values of $\xi$ the two Majorana fermions merge and behave as a single Dirac fermion while for small values of $\xi$ the low-energy physics is essentially governed by Majorana degrees of freedom. The effective low-energy Hamiltonian of the topological superconductor is

$$
\hat{H}_{t s c}=\frac{\mathrm{i}}{2} \xi \gamma_{2} \gamma_{1}
$$

where $\gamma_{1,2}$ are the Majorana fermion annihilation operators identical to the corresponding creation operators, $\gamma_{1,2}^{\dagger}=\gamma_{1,2}$. These annihilation operators satisfy the anticommutation relations $\left\{\gamma_{i}, \gamma_{j}\right\}=2 \delta_{i j}$ implying that the associative algebra generated by the set $\left\{1, \gamma_{1}, \gamma_{2}\right\}$ is the Clifford algebra 48].

The quantum dot interacts with the topological superconductor via tunneling involving only the Majorana mode $\gamma_{1}$ as has been suggested in numerous literature (see, e.g., Refs. [15, 27, 28, 44]),

$$
\hat{H}_{d-t s c}=\eta^{*} d^{\dagger} \gamma_{1}+\text { H.c. }
$$

where the absolute value $|\eta|$ of the tunneling matrix element characterizes the strength of the tunneling between the quantum dot and the topological superconductor.

To explore nonlinear thermoelectric response of the system specified by the Hamiltonian

$$
\hat{H}=\hat{H}_{d}+\hat{H}_{c}+\hat{H}_{d-c}+\hat{H}_{t s c}+\hat{H}_{d-t s c}
$$

it is convenient to resort to the Keldysh field integral framework [49, 50] as a general tool providing various correlation functions in a simple systematic way. To this end we write down the source dependent Keldysh generating functional:

$$
Z\left[J_{l}(t)\right]=\int \mathcal{D}[\bar{\theta}(t), \theta(t)] e^{\frac{\mathrm{i}}{\hbar} S_{K}\left[\bar{\theta}(t), \theta(t) ; J_{l}(t)\right]},
$$

where $\{\bar{\theta}(t), \theta(t)\}=\left\{\bar{\psi}(t), \psi(t) ; \bar{\phi}_{l k}(t), \phi_{l k}(t) ; \bar{\zeta}(t), \zeta(t)\right\}$ is the set of the Grassmann fields of, respectively, the quantum dot, contacts and topological superconductor while $J_{l}(t)$ is the source field. Note, the fundamental normalization $Z\left[J_{l}(t)=0\right]=1$. In Eq. (8) the time argument $t$ runs over the Keldysh closed time contour $C_{K}, t \in C_{K}$.

The total Keldysh action $S_{K}\left[\bar{\theta}(t), \theta(t) ; J_{l}(t)\right]$ consists of the Keldysh actions of the isolated quantum dot, contacts and topological superconductor, the tunneling actions describing the interaction of the quantum dot with the contacts and topological superconductor as well as the source action.

The Keldysh actions of the isolated quantum dot, $S_{d}[\bar{\psi}(t), \psi(t)]$, contacts, $S_{c}\left[\bar{\phi}_{l k}(t), \phi_{l k}(t)\right]$, and topological superconductor, $S_{t s c}[\bar{\zeta}(t), \zeta(t)]$, have the conventional form of upper triangular $2 \times 2$ matrices in the retardedadvanced space with the upper/lower diagonal elements representing the inverse retarded/advanced Green's functions of the corresponding isolated system while the upper off-diagonal elements of these matrices have the form i $\delta(1-2 f)$ where $\delta \rightarrow 0^{+}$and $f$ is the corresponding Fermi-Dirac distribution which, e.g., for the contacts is given by Eq. (2).

The tunneling actions describing the interactions of the quantum dot with the contacts and topological superconductor are, respectively, given as

$$
\begin{aligned}
& S_{d-c}\left[\bar{\psi}(t), \psi(t) ; \bar{\phi}_{l k}(t), \phi_{l k}(t)\right]= \\
& =-\int_{-\infty}^{\infty} d t \sum_{l=\{L, R\}} \sum_{k}\left\{T _ { l } \left[\bar{\phi}_{l k+}(t) \psi_{+}(t)-\right.\right. \\
& \left.\left.-\bar{\phi}_{l k-}(t) \psi_{-}(t)\right]+ \text { G.c. }\right\}, \\
& S_{d-t s c}[\bar{\psi}(t), \psi(t) ; \bar{\zeta}(t), \zeta(t)]= \\
& =-\int_{-\infty}^{\infty} d t\left\{\eta ^ { * } \left[\bar{\psi}_{+}(t) \zeta_{+}(t)+\bar{\psi}_{+}(t) \bar{\zeta}_{+}(t)-\right.\right. \\
& \left.\left.-\bar{\psi}_{-}(t) \zeta_{-}(t)-\bar{\psi}_{-}(t) \bar{\zeta}_{-}(t)\right]+ \text { G.c. }\right\},
\end{aligned}
$$

where in the right hand side the time argument $t$ runs over the real axis and the subindex $+/-$ denotes the forward/backward branches of the Keldysh closed time contour. The abbreviation G.c. stands for the Grassmann conjugation, the generalization of the Hermitian conjugation for the case when Grassmann variables are involved in a mathematical expression.

Finally, the source action is

$$
\begin{aligned}
& S_{s c r}\left[\bar{\psi}(t), \psi(t) ; \bar{\phi}_{l k}(t), \phi_{l k}(t) ; J_{l}(t)\right]= \\
& =-\int_{-\infty}^{\infty} d t \sum_{l=\{L, R\}} \sum_{q=\{+,-\}} J_{l q}(t) I_{l q}(t),
\end{aligned}
$$

where $I_{l q}(t)$ is the field representing the electric current in the left $(l=L)$ or right $(l=R)$ contact on the forward $(q=+)$ or backward $(q=-)$ branch of the Keldysh closed time contour:

$$
I_{l q}(t) \equiv \frac{\mathrm{i} e}{\hbar} \sum_{k}\left[T_{l} \bar{\phi}_{l k q}(t) \psi_{q}(t)-\text { G.c. }\right] \text {. }
$$

Using the total Keldysh action,

$$
\begin{aligned}
& S_{K}\left[\bar{\theta}(t), \theta(t) ; J_{l}(t)\right]= \\
& =S_{d}[\bar{\psi}(t), \psi(t)]+S_{c}\left[\bar{\phi}_{l k}(t), \phi_{l k}(t)\right]+ \\
& +S_{t s c}[\bar{\zeta}(t), \zeta(t)]+S_{d-c}\left[\bar{\psi}(t), \psi(t) ; \bar{\phi}_{l k}(t), \phi_{l k}(t)\right]+ \\
& +S_{d-t s c}[\bar{\psi}(t), \psi(t) ; \bar{\zeta}(t), \zeta(t)]+ \\
& +S_{s c r}\left[\bar{\psi}(t), \psi(t) ; \bar{\phi}_{l k}(t), \phi_{l k}(t) ; J_{l}(t)\right]
\end{aligned}
$$


one can easily obtain the mean electric current in the left $(l=L)$ or right $(l=R)$ contact by taking the first derivative of $Z\left[J_{l}(t)\right]$ with respect to the corresponding source field:

$$
\left\langle I_{l}\right\rangle \equiv\left\langle I_{l q}(t)\right\rangle_{S_{K}}=\left.\mathrm{i} \hbar \frac{\delta Z\left[J_{l}(t)\right]}{\delta J_{l q}(t)}\right|_{J_{l q}(t)=0},
$$

where the angular brackets $\langle\cdots\rangle_{S_{K}}$ denote the average of a functional of Grassmann fields with respect to the total Keldysh action (13) taken at zero source field,

$$
\begin{aligned}
& \langle\mathcal{F}[\bar{\theta}(t), \theta(t)]\rangle_{S_{K}} \equiv \\
& \equiv \int \mathcal{D}[\bar{\theta}(\tilde{t}), \theta(\tilde{t})] e^{\frac{i}{\hbar} S_{K}[\bar{\theta}(\tilde{t}), \theta(\tilde{t})]} \mathcal{F}[\bar{\theta}(t), \theta(t)], \\
& S_{K}[\bar{\theta}(t), \theta(t)] \equiv S_{K}\left[\bar{\theta}(t), \theta(t) ; J_{l}(t)=0\right]= \\
& =S_{d}[\bar{\psi}(t), \psi(t)]+S_{c}\left[\bar{\phi}_{l k}(t), \phi_{l k}(t)\right]+ \\
& +S_{t s c}[\bar{\zeta}(t), \zeta(t)]+S_{d-c}\left[\bar{\psi}(t), \psi(t) ; \bar{\phi}_{l k}(t), \phi_{l k}(t)\right]+ \\
& +S_{d-t s c}[\bar{\psi}(t), \psi(t) ; \bar{\zeta}(t), \zeta(t)] .
\end{aligned}
$$

The values of $q$ and $t$ in Eq. (14) are arbitrary since the final result does not depend on the choice of a branch of the Keldysh closed time contour as well as on the choice of an instant of time on that branch because we consider only stationary nonequilibrium states. It is straightforward to verify that Eq. 14 leads to the Meir-Wingreen result [51].

In a similar way one can obtain the current-current correlator by taking the second derivative of $Z\left[J_{l}(t)\right]$ with respect to the corresponding source fields:

$$
\begin{aligned}
& \left\langle I_{l}(t) I_{l^{\prime}}\left(t^{\prime}\right)\right\rangle \equiv\left\langle I_{l-}(t) I_{l^{\prime}+}\left(t^{\prime}\right)\right\rangle_{S_{K}}= \\
& =\left.(\mathrm{i} \hbar)^{2} \frac{\delta^{2} Z\left[J_{l}(t)\right]}{\delta J_{l-}(t) \delta J_{l^{\prime}+}\left(t^{\prime}\right)}\right|_{J_{l q}(t)=0} .
\end{aligned}
$$

The technical steps one makes in the calculation of the second derivative in Eq. 17) are mainly straightforward. One should only take proper care of the fact that due to the presence of the topological superconductor there will appear anomalous contributions when averaging products of four Grassmann fields of the quantum dot. To facilitate one's derivation of the anomalous terms and to avoid technicalities in the main text we provide the main details relevant for the calculation of the second derivative in Eq. (17) in the appendix.

The first and second derivatives of the Keldysh generating functional are enough to explore nonlinear Majorana thermoelectric response of the system in terms of the mean value and fluctuations of the electric current.

\section{UNIVERSAL MAJORANA THERMOELECTRIC TRANSPORT}

For definiteness below we focus on transport measurements in the left $(L)$ contact which is assumed to be hot. The temperature difference $\Delta T$ between the contacts may be parameterized by a thermal voltage $V_{T}$,

$$
V_{T} \equiv \frac{k_{\mathrm{B}} \Delta T}{e}
$$

where $k_{\mathrm{B}}$ is the Boltzmann constant.

Additionally, we will consider the situation when the quantum dot couples symmetrically to the left and right contacts, $\Gamma_{L}=\Gamma_{R}=\Gamma / 2$.

To solely focus on universal Majorana physics we explore the regime dominated by the Majorana tunneling,

$$
|\eta|>\max \left\{\left|\epsilon_{d}\right|,|e V|, e V_{T}, k_{\mathrm{B}} T, \Gamma, \xi\right\} .
$$

Here $\epsilon_{d}$ may be tuned by an external gate voltage to satisfy 19 . Similarly, the bias voltage $V$, thermal voltage $V_{T}$ and temperature $T$ may be externally adjusted to satisfy 19 . The Majorana overlap $\xi$ will be small if the topological superconductor in the setup is chosen to be long enough so that the two Majorana fermions do not merge into a single Dirac fermion during the tunneling into the quantum dot. In modern experiments 52 the values of $|\eta|$ and $\Gamma$ are readily controlled via external gates whose potentials can increase or decrease the height of the potential barriers between the quantum dot and contacts in order to vary $\Gamma$ as well as between the quantum dot and topological superconductor in order to vary $|\eta|$. In this way in modern laboratories $|\eta|$ may be increased while $\Gamma$ may be decreased in order to reach the condition in (19). Thus, besides its theoretical importance, the Majorana transport regime, specified by 19 , is of particular experimental interest.

Before we start to discuss our results it is important to mention how we obtain various nonlinear response coefficients. In what follows we use the formalism presented in Section II and the appendix and perform numerical calculations of corresponding integrals in the energy domain to obtain the mean electric current and current-current correlations. This formalism allows one to compute not only the mean electric current and current-current correlations but also various derivatives of these quantities. The calculations of the derivatives may be performed in two different ways. On one side, one can calculate derivatives using finite differences. In this case one computes the mean electric current and current-current correlations on a fine grid of the bias voltage and thermal voltage and after that applies conventional finite difference schemes for the first and second derivatives. On the other side, one notices (see the appendix) that the dependence of both the mean electric current and current-current correlations on the bias voltage and thermal voltage enters through the Keldysh components of the Green's functions, more specifically, through the Fermi-Dirac distributions 2. Therefore, one can first calculate various derivatives of corresponding integrands. This simply reduces to analytical differentiations of the Fermi-Dirac distributions (2). After that one performs numerical integration of these differentiated integrands which now involve various analytical derivatives of the Fermi-Dirac 


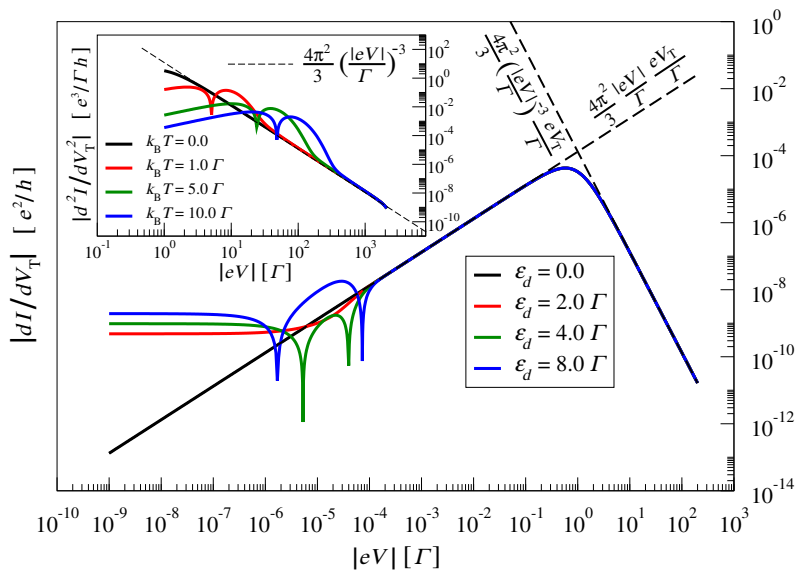

FIG. 2. The magnitude of the first derivative of the mean electric current with respect to the thermal voltage, $\left|\partial I\left(V, V_{T}\right) / \partial V_{T}\right|$, as a function of the bias voltage $V$ at $T=0$, $e V_{T} / \Gamma=10^{-5}$ and for different gate voltages, specified by the quantum dot single-particle energy level $\epsilon_{d}$. The Majorana fermions overlap weakly, $\xi / \Gamma=10^{-4}$. The strength of the Majorana tunneling is $|\eta| / \Gamma=10^{3}$. Here the dips in the nonuniversal $(|e V| \lesssim \xi)$ behavior of the curves with $\epsilon_{d} / \Gamma=4.0$ and $\epsilon_{d} / \Gamma=8.0$ correspond to those values of the bias voltage where $\partial I\left(V, V_{T}\right) / \partial V_{T}=0$, that is to those values of $V$ where $\partial I\left(V, V_{T}\right) / \partial V_{T}$ changes its sign. The inset shows the robustness of the universal (independent of $\epsilon_{d}$ ) behavior of the second derivative of the mean electric current with respect to the thermal voltage at large bias voltages when the temperature increases. At higher temperatures there are values of the bias voltage where $\partial^{2} I\left(V, V_{T}\right) / \partial V_{T}^{2}=0$, that is points where $\partial^{2} I\left(V, V_{T}\right) / \partial V_{T}^{2}$ changes its sign. Since we use the logarithmic scale, we plot the magnitude of the second derivative, $\left|\partial^{2} I\left(V, V_{T}\right) / \partial V_{T}^{2}\right|$, which displays dips at its zeroes.

distributions (2). We find that in all of our calculations these two ways of computing derivatives give the same results within a good numerical precision. This is a very good test that our numerical results are reliable. However, in order to reach higher precision to obtain analytical expressions, which will be discussed below, we prefer to use the second way involving analytical differentiations of the Fermi-Dirac distributions (2).

Another important aspect concerns the analytical asymptotic limits presented below. They are obtained by inspection of our numerical results. We will use the term asymptotic limit for an analytical expression if our numerical results reproduce this analytical expression with any desired numerical precision by adjusting the physical parameters of the system to satisfy the inequalities, specifying the regime of applicability of this analytical expression, with any desired degree of accuracy. In more mathematical terms, referring to an analytical expression as an asymptotic limit means that the stronger the inequalities, specifying the regime of applicability of this analytical expression, are fulfilled the more digits after the decimal point in our numerical results reproduce this analytical expression. In other words, for the theoretical model presented in Section II the asymptotic limits presented below are analytical expressions to which numerical results converge when one gradually increases the numerical precision and no further approximation to the theoretical model in Section [1 is assumed.

Finally, we would like to specify what we understand under universality of our results which will be presented below. We will call a quantity universal if this quantity is independent of the parameters characterizing exclusively the quantum dot. In our case such a parameter is $\epsilon_{d}$ which is tuned by an external gate voltage. This is experimentally relevant because in a realistic experiment one may easily vary the gate voltage and observe which transport quantities do not change in response to this variation of the gate voltage. Similar universality happens in the Kondo effect [47] where universality is understood as independence of response coefficients on $\epsilon_{d}$ which enters only through the Kondo temperature $T_{K}$. The difference between the Majorana universality and Kondo universality is in the scaling. For the present case of the Majorana universality the scaling is given by $\Gamma$ whereas for the Kondo universality it is given by the Kondo temperature $T_{K}$ which is also a function of $\Gamma$. In all of our calculations presented below we use $\epsilon_{d}>0$. More specifically, to perform concrete calculations we put $\epsilon_{d}=8 \Gamma$ but universal results do not change if one uses other values for $\epsilon_{d}$ satisfying the condition in 19 . Using positive values for $\epsilon_{d}$ one puts the quantum dot in the empty orbital regime [47. As a result, in a realistic experiment the Kondo effect is switched off and one observes only the Majorana universality. Note, that in the absence of the Majorana bound states one would observe strong dependence of response coefficients on $\epsilon_{d}$ for bias voltages $|e V| \sim \epsilon_{d}$. Therefore, observing independence of transport quantities on the gate voltage for any bias voltage already provides information about signatures of unpaired Majorana fermions. Of course, such a quantity, which is universal in the above sense, may depend on the tunneling coupling to the contacts, $\Gamma$, or on the tunneling coupling to the topological superconductor, $\eta$, or on both. Thus the universality understood in the above sense is only a qualitative Majorana signature but not quantitative. For example, a response coefficient may have a universal asymptotic behavior at high bias voltages. Let us assume that this universal asymptotic behavior is given by a certain universal (independent of $\left.\epsilon_{d}\right)$ function of $e V / \Gamma$. Although the universality of this function is a qualitative signature of unpaired Majorana fermions, the values of the coefficients in the expansion of this universal function in powers of $e V / \Gamma$ will depend on the definition of $\Gamma$ and cannot serve as a quantitative signature of unpaired Majorana fermions. To provide quantitative signatures of unpaired Majorana fermions we define truly universal quantities. We will call a quantity truly universal if this quantity does not depend on both the parameters of the quantum dot, such as $\epsilon_{d}$, and the tunneling couplings to the contacts, $\Gamma$, as well as to the topological superconductor, $\eta$. For example, if there are two universal quantities, the ratio between these two 


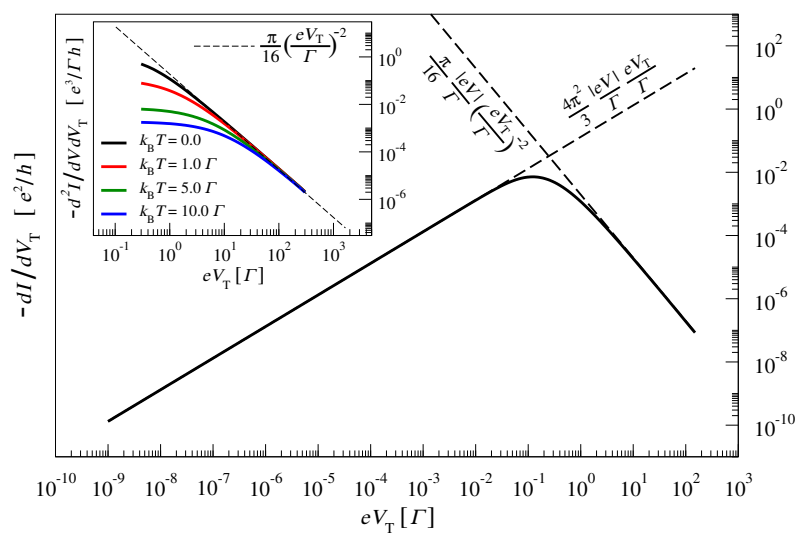

FIG. 3. The first derivative of the mean current with respect to the thermal voltage, $\partial I\left(V, V_{T}\right) / \partial V_{T}$, as a universal (independent of $\epsilon_{d}$ ) function of the thermal voltage $V_{T}$ at $T=0$, $|e V| / \Gamma=10^{-2}$. The other parameters have the same values as in Fig. 2. The inset shows the robustness of the universal (independent of $\epsilon_{d}$ ) behavior of the second mixed derivative $\partial^{2} I\left(V, V_{T}\right) / \partial V \partial V_{T}$ at large thermal voltages when the temperature increases.

quantities may be truly universal because $\Gamma$ and $\eta$ may cancel out in this ratio which therefore becomes a unique quantitative signature of unpaired Majorana fermions. Below in the discussion of our results we will always distinguish between universal and truly universal results.

\section{A. Nonequilibrium response of the mean thermoelectric current}

Let us first examine the universal Majorana signatures one can detect in the thermoelectric response of the mean electric current $I\left(V, V_{T}\right)$ as a function of the bias voltage and thermal voltage. It is obtained from Eq. (14) with $l=L$, that is $I\left(V, V_{T}\right) \equiv\left\langle I_{L}\right\rangle$.

In Fig. 2 we show the magnitude of the thermoelectric coefficient representing the first derivative of the mean electric current with respect to the thermal voltage, $\left|\partial I\left(V, V_{T}\right) / \partial V_{T}\right|$, as a function of the bias voltage $V$ at zero temperature for the case $e V_{T} \ll \Gamma$ and for different gate voltages parameterized by the values of $\epsilon_{d}$. One can see that despite the fact that the Majorana fermions are well separated $\left(\xi / \Gamma=10^{-4}\right)$ and the Majorana tunneling is strong $\left(|\eta| / \Gamma=10^{3}\right)$, the curves do not collapse on a single universal (independent of $\epsilon_{d}$ ) curve. We find that only for $|e V| \gg \xi$ the curves do collapse on a single universal curve whose shape does not depend on the gate voltage which regulates the value of $\epsilon_{d}$. As mentioned in Section [II. we show only the case $\epsilon_{d}>0$ since universal Majorana signatures do not depend on both the magnitude and sign of $\epsilon_{d}$. At the same time for $\epsilon_{d}>0$ the quantum dot is in the empty orbital regime which excludes the Kondo resonance [4] from the physical setup. Thus we conclude that the linear thermoelectric response, i.e. response at small bias voltages $(|e V| \ll \xi)$, of the mean electric current provides only non-universal Majorana signatures and, therefore, it does not represent any interest as a unique universal signature of Majorana fermions.

To access the universal Majorana physics in the mean thermoelectric quantities one has to resort to the nonlinear $(|e V|>\xi)$ thermoelectric response. In this universal regime we find the following asymptotic limits for the thermoelectric coefficient $\partial I\left(V, V_{T}\right) / \partial V_{T}$ :

$$
\frac{\partial I\left(V, V_{T}\right)}{\partial V_{T}}=-\frac{e^{2}}{h} \frac{4 \pi^{2}}{3} \frac{|e V|}{\Gamma} \frac{e V_{T}}{\Gamma}
$$

for

$$
|\eta| \gg \Gamma \gg\left(|e V|, e V_{T}\right), \quad|e V| \gg \xi
$$

and

$$
\frac{\partial I\left(V, V_{T}\right)}{\partial V_{T}}=-\frac{e^{2}}{h} \frac{4 \pi^{2}}{3}\left(\frac{|e V|}{\Gamma}\right)^{-3} \frac{e V_{T}}{\Gamma}
$$

for

$$
|\eta| \gg|e V| \gg \Gamma \gg e V_{T}, \quad \Gamma \gg \xi
$$

The universal laws given by Eqs. 20 and 22 are depicted by dashed lines in Fig. 2 .

The inset in Fig. 2 shows the universal (independent of $\epsilon_{d}$ ) Majorana behavior of the thermoelectric coefficient representing the second derivative of the mean electric current with respect to the thermal voltage, $\partial^{2} I\left(V, V_{T}\right) / \partial V_{T}^{2}$, as a function of the bias voltage $V$ in the high-energy regime specified by the inequality in 23. This universal Majorana high-energy behavior is independent of the thermal voltage $V_{T}$. As one can see, the second derivative $\partial^{2} I\left(V, V_{T}\right) / \partial V_{T}^{2}$ is very robust with respect to high temperatures. At high bias voltages it is given by the asymptotic limit,

$$
\frac{\partial^{2} I\left(V, V_{T}\right)}{\partial V_{T}^{2}}=-\frac{e^{3}}{\Gamma h} \frac{4 \pi^{2}}{3}\left(\frac{|e V|}{\Gamma}\right)^{-3},
$$

shown as the dashed line in the inset in Fig. 2. The expression in Eq. (24) follows from Eq. (22) if the inequality in (23) is fulfilled. The universal behavior of $\partial^{2} I\left(V, V_{T}\right) / \partial V_{T}^{2}$ given by Eq. 24 is almost unchanged for $|e V| \gg k_{\mathrm{B}} T$ when the temperature increases up to $k_{\mathrm{B}} T \sim 10 \Gamma$, or in units of $|\eta|, k_{\mathrm{B}} T \sim 0.01|\eta|$.

As it follows from the above analysis, to focus on universal thermoelectric Majorana signatures in the mean electric current one has to apply bias voltages $|\mathrm{eV}| \gg \xi$. In Fig. 3 we show the universal Majorana behavior of the thermoelectric coefficient representing the first derivative of the mean electric current with respect to the thermal voltage, $\partial I\left(V, V_{T}\right) / \partial V_{T}$, as a function of the thermal voltage $V_{T}$ at zero temperature and at a fixed value of the bias voltage $\Gamma \gg|e V| \gg \xi$. For the particular example shown in Fig. 3 we put $|e V| / \xi=100,|e V| / \Gamma=0.01$. As a result, the curve in Fig. 3 is universal in the whole 


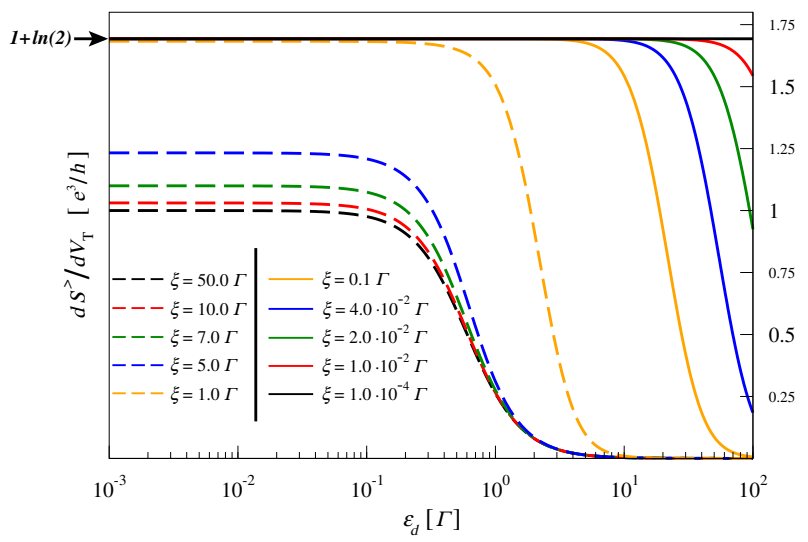

FIG. 4. The first derivative of the greater currentcurrent correlator with respect to the thermal voltage, $\partial S^{>}\left(V, V_{T}\right) / \partial V_{T}$, as a function of the gate voltage, specified by the quantum dot single-particle energy level $\epsilon_{d}$, for different values of the Majorana overlap energy $\xi$. Here the parameters are as follows $T=0, V=0, e V_{T} / \Gamma=10^{-6}$ and $\eta / \Gamma=10^{3}$.

range of the thermal voltage. The universal behavior of $\partial I\left(V, V_{T}\right) / \partial V_{T}$ as a function of the thermal voltage $V_{T}$ in the regime specified by the inequality $(21)$ is given by the asymptotic limit, Eq. (20), shown in Fig. 3 as the dashed line with a positive slope. However, for

$$
|\eta| \gg e V_{T} \gg \Gamma \gg|e V| \gg \xi
$$

we find the following universal asymptotic limit:

$$
\frac{\partial I\left(V, V_{T}\right)}{\partial V_{T}}=-\frac{e^{2}}{h} \frac{\pi}{16} \frac{|e V|}{\Gamma}\left(\frac{e V_{T}}{\Gamma}\right)^{-2}
$$

which is shown in Fig. 3 as the dashed line with a negative slope.

The inset in Fig. 3 shows the universal Majorana behavior of the thermoelectric coefficient representing the second mixed derivative of the mean electric current with respect to the thermal voltage and the bias voltage $\partial^{2} I\left(V, V_{T}\right) / \partial V \partial V_{T}$, as a function of the thermal voltage $V_{T}$ in the high-energy regime specified by the inequality in 25). The universal Majorana high-energy behavior of $\partial^{2} I\left(V, V_{T}\right) / \partial V \partial V_{T}$ is independent of the bias voltage $V$. Like the second derivative $\partial^{2} I\left(V, V_{T}\right) / \partial V_{T}^{2}$ as a function of the bias voltage $V$, the second mixed derivative $\partial^{2} I\left(V, V_{T}\right) / \partial V \partial V_{T}$ as a function of the thermal voltage $V_{T}$ is robust with respect to high temperatures. At high thermal voltages it is given by the asymptotic limit obtained from Eq. (26) if the inequality in (25) is fulfilled. Its universal behavior is shown by the dashed line in the inset in Fig. 3. It is protected by high thermal voltages $e V_{T} \gg k_{\mathrm{B}} T$ from thermal destruction effects as shown in the inset for high temperatures, $k_{\mathrm{B}} T \sim 10 \Gamma$ $\left(k_{\mathrm{B}} T \sim 0.01|\eta|\right)$.

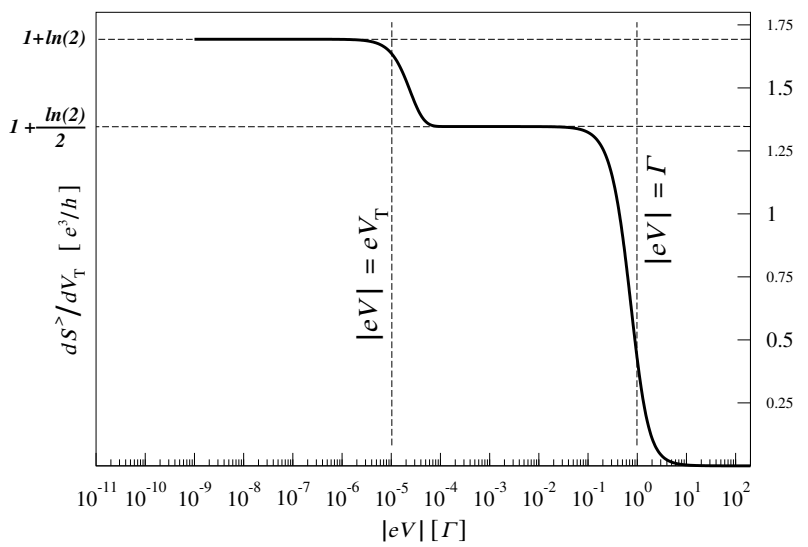

FIG. 5. The first derivative of the greater currentcurrent correlator with respect to the thermal voltage, $\partial S^{>}\left(V, V_{T}\right) / \partial V_{T}$, as a universal (independent of $\epsilon_{d}$ ) function of the bias voltage $V$ at $T=0, e V_{T} / \Gamma=10^{-5}$. The Majorana overlap energy and the strength of the Majorana tunneling are $\xi / \Gamma=10^{-4}$ and $|\eta| / \Gamma=10^{3}$, respectively.

\section{B. Thermoelectric fluctuations beyond linear response}

Now we address the universal Majorana signatures present in the thermoelectric response of the fluctuations of the electric current. To this end we use Eq. (17) with $l=l^{\prime}=L$ to obtain the current-current correlator $\left\langle I_{L}(t) I_{L}\left(t^{\prime}\right)\right\rangle$. Finite deviations of the electric current from its mean value, $\delta I_{L}(t)=I_{L}(t)-I\left(V, V_{T}\right)$, may be characterized via the greater current-current correlator defined as $S^{>}\left(t, t^{\prime} ; V, V_{T}\right) \equiv\left\langle\delta I_{L}(t) \delta I_{L}\left(t^{\prime}\right)\right\rangle$. It can be expressed through the correlator $\left\langle I_{L}(t) I_{L}\left(t^{\prime}\right)\right\rangle$ as follows:

$$
S^{>}\left(t, t^{\prime} ; V, V_{T}\right)=\left\langle I_{L}(t) I_{L}\left(t^{\prime}\right)\right\rangle-I^{2}\left(V, V_{T}\right) .
$$

Due to the stationary nonequilibrium $S^{>}\left(t, t^{\prime} ; V, V_{T}\right)=$ $S^{>}\left(t-t^{\prime} ; V, V_{T}\right)$ and the physical quantity measured experimentally is the Fourier transform

$$
S^{>}\left(\omega ; V, V_{T}\right)=\int_{-\infty}^{\infty} d t e^{\mathrm{i} \omega t} S^{>}\left(t ; V, V_{T}\right) .
$$

Below we focus on the zero frequency noise as a function of the bias voltage and thermal voltage,

$$
S^{>}\left(V, V_{T}\right) \equiv S^{>}\left(\omega=0 ; V, V_{T}\right) .
$$

As we know from the previous subsection the mean Majorana thermoelectric response becomes universal (independent of $\epsilon_{d}$ ) only if the bias voltage is large enough, $|e V| \gg \xi$. The first question is thus whether the same happens with the Majorana thermoelectric response of the fluctuations of the electric current. We find that, in contrast to the mean Majorana thermoelectric response, the fluctuations of the electric current are universal in the whole range of the bias voltage. This means that the Majorana thermoelectric response of the electric current 


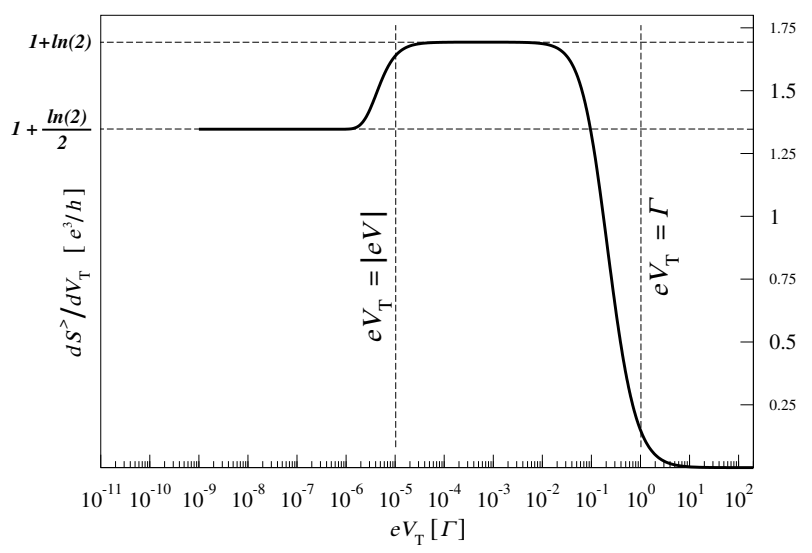

FIG. 6. The first derivative of the greater currentcurrent correlator with respect to the thermal voltage, $\partial S^{>}\left(V, V_{T}\right) / \partial V_{T}$, as a universal (independent of $\epsilon_{d}$ ) function of the thermal voltage $V_{T}$ at $T=0,|e V| / \Gamma=10^{-5}$. The Majorana overlap energy and the strength of the Majorana tunneling are $\xi / \Gamma=10^{-4}$ and $|\eta| / \Gamma=10^{3}$, respectively.

noise is independent of $\epsilon_{d}$ at any bias voltage $V$. In Fig. 4 we show the thermoelectric coefficient representing the first derivative of the greater noise with respect to the thermal voltage, $\partial S^{>}\left(V, V_{T}\right) / \partial V_{T}$, as a function of the gate voltage, parameterized by the value of $\epsilon_{d}$, at zero temperature, zero bias voltage, $e V_{T} \ll \Gamma$ and different values of the Majorana overlap energy $\xi$ in the regime specified in (19) where the Majorana tunneling dominates. As can be seen, for large values of the Majorana overlap energy there is a very strong dependence on the gate voltage with the unit plateau at small gate voltages. When $\xi$ decreases the value of the plateau grows and the plateau becomes wider. Finally, when $\xi$ is very small (the same as in the previous subsection on the mean electric current, $\xi / \Gamma=10^{-4}$ ) the Majorana fermions overlap very weakly. In this situation the value of the plateau saturates at the asymptotic limit $\left(e^{3} / h\right)[1+\ln (2)]$ and the width of the plateau gets extremely wide. Therefore, when the Majorana tunneling is strong enough, so that $(19)$ is satisfied, the fluctuation thermoelectric coefficient $\partial S^{>}\left(V, V_{T}\right) / \partial V_{T}$ becomes independent of $\epsilon_{d}, \Gamma$ and $\eta$, that is it becomes truly universal with the value $\left(e^{3} / h\right)[1+\ln (2)]$ (the black horizontal line in Fig. 4).

The universal Majorana behavior of the fluctuation thermoelectric coefficient $\partial S^{>}\left(V, V_{T}\right) / \partial V_{T}$ as a function of the bias voltage $V$ is shown in Fig. 5 at zero temperature and $e V_{T} \ll \Gamma$. The values of $\xi$ and $|\eta|$ are the same as for the mean electric current from the previous subsection so that the Majorana overlap is very weak and the Majorana tunneling is very strong. For

$$
|\eta| \gg \Gamma \gg e V_{T} \gg|e V|, \quad \Gamma \gg \xi
$$

it turns out that the fluctuation thermoelectric coefficient $\partial S^{>}\left(V, V_{T}\right) / \partial V_{T}$ does not depend on $V$ and is truly universal (independent of $\epsilon_{d}, \Gamma$ and $\eta$ ). The asymptotic limit

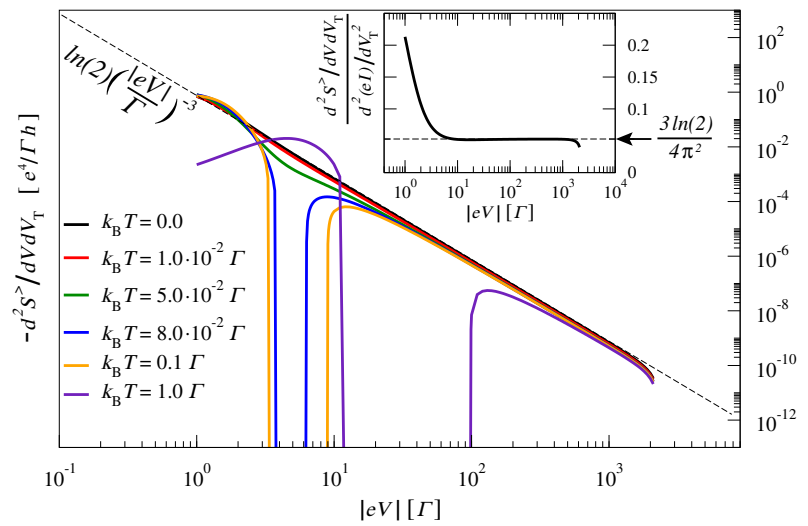

FIG. 7. The universal (independent of $\epsilon_{d}$ ) behavior of the second mixed derivative of the greater current-current correlator with respect to the thermal voltage and the bias voltage, $\partial^{2} S^{>}\left(V, V_{T}\right) / \partial V \partial V_{T}$, at large bias voltages $V$. The thermal voltage is $e V_{T} / \Gamma=10^{-2}$. The Majorana overlap energy and the strength of the Majorana tunneling are $\xi / \Gamma=10^{-4}$ and $|\eta| / \Gamma=10^{3}$, respectively. The curves demonstrate robustness of $\partial^{2} S^{>}\left(V, V_{T}\right) / \partial V \partial V_{T}$ at $|e V| \gg k_{\mathrm{B}} T$ when the temperature increases. The inset shows the universal nonlinear response thermoelectric ratio of the second mixed derivative of the greater current-current correlator with respect to the thermal voltage and the bias voltage to the second derivative of the mean electric current times the electron charge with respect to the thermal voltage, $\left\{\partial^{2} S^{>}\left(V, V_{T}\right) / \partial V \partial V_{T}\right\} /\left\{\partial^{2}\left[e I\left(V, V_{T}\right)\right] / \partial V_{T}^{2}\right\}$, at $T=0$.

of this truly universal constant is

$$
\frac{\partial S^{>}\left(V, V_{T}\right)}{\partial V_{T}}=\frac{e^{3}}{h}[1+\ln (2)] .
$$

For

$$
|\eta| \gg \Gamma \gg|e V| \gg e V_{T}, \quad \Gamma \gg \xi
$$

the fluctuation thermoelectric coefficient $\partial S^{>}\left(V, V_{T}\right) / \partial V_{T}$ does not depend on $V$ either and is equal to a different truly universal constant. The asymptotic limit in this case is

$$
\frac{\partial S^{>}\left(V, V_{T}\right)}{\partial V_{T}}=\frac{e^{3}}{h}\left[1+\frac{1}{2} \ln (2)\right]
$$

These two plateaus are explicitly visible in Fig. 5.

In the high-energy regime specified in (23) the universal asymptotic limit of the fluctuation thermoelectric coefficient $\partial S^{>}\left(V, V_{T}\right) / \partial V_{T}$ is

$$
\frac{\partial S^{>}\left(V, V_{T}\right)}{\partial V_{T}}=\frac{e^{3}}{h} \frac{\ln (2)}{2}\left(\frac{|e V|}{\Gamma}\right)^{-2}
$$

As a function of the thermal voltage $V_{T}$ the fluctuation thermoelectric coefficient $\partial S^{>}\left(V, V_{T}\right) / \partial V_{T}$ is shown in Fig. 6. In the regimes specified in (30) and 32 this dependence has, respectively, the plateaus (31) and (33) with the only difference in the order of these plateaus. 


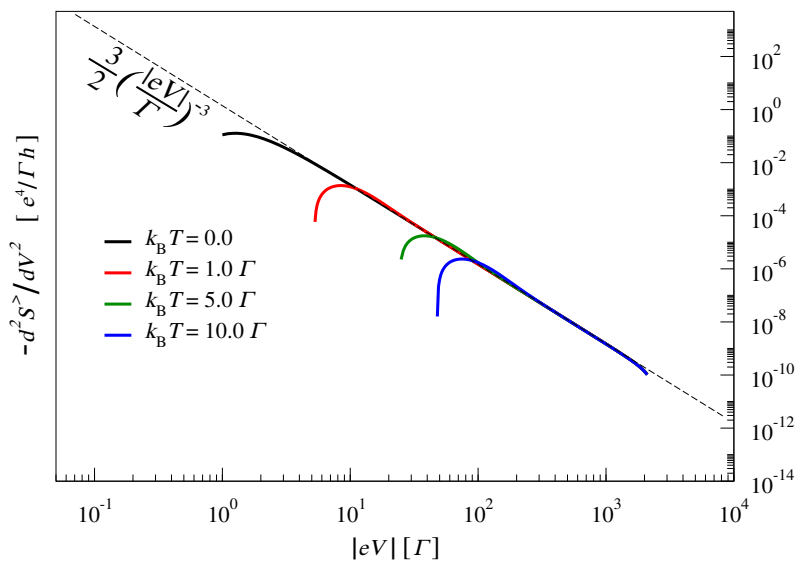

FIG. 8. The universal (independent of $\epsilon_{d}$ ) behavior of the second derivative of the greater current-current correlator with respect to the bias voltage, $\partial^{2} S^{>}\left(V, V_{T}\right) / \partial V^{2}$, at large bias voltages $V$. The thermal voltage is $e V_{T} / \Gamma=10^{-5}$. The Majorana overlap energy and the strength of the Majorana tunneling are $\xi / \Gamma=10^{-4}$ and $|\eta| / \Gamma=10^{3}$, respectively. The curves are shown for different temperatures and demonstrate remarkable robustness of $\partial^{2} S^{>}\left(V, V_{T}\right) / \partial V^{2}$ at $|e V| \gg k_{\mathrm{B}} T$ when the temperature increases.

Here the lowest plateau (33) is followed by the highest one 31.

The physical mechanism underlying the fact that the truly universal plateau (31) in the regime 30 is larger than the truly universal plateau (33) in the regime (32) can be understood as follows. As schematically shown in Fig. 1, at $T=0$ the Fermi-Dirac distribution in the left (hot) contact is smeared over the energy range $e V_{T}$ around the Fermi energy of this contact. When we have $e V_{T} \gg|e V|$, there exist two electron flows. The first flow, from the right (cold) contact to the left contact, is induced by electrons coming from the filled states below the Fermi energy of the right contact to the partially filled states below and above the Fermi energy of the left contact. The second flow, from the left contact to the right contact, is induced by electrons coming from the partially filled states above the Fermi energy of the left contact to the empty states above the Fermi energy of the right contact. Both of these electron flows fluctuate and contribute to the total noise $S^{>}\left(V, V_{T}\right)$ which grows linearly as a function of $V_{T}$ with the slope given by Eq. (31). However, when we decrease the thermal voltage up to the bias voltage, $e V_{T} \sim|e V|$, the second flow significantly decays and becomes negligible for $e V_{T} \ll|e V|$ because the population of the states in the left contact above its Fermi energy rapidly goes to zero. In this situation the noise contribution from the second flow vanishes and only fluctuations of the first flow essentially contribute to the total noise $S^{>}\left(V, V_{T}\right)$ which still grows linearly as a function of $V_{T}$ but with the reduced slope given by Eq. (33).

In the high-energy regime,

$$
|\eta| \gg e V_{T} \gg \Gamma \gg|e V|, \Gamma \gg \xi,
$$

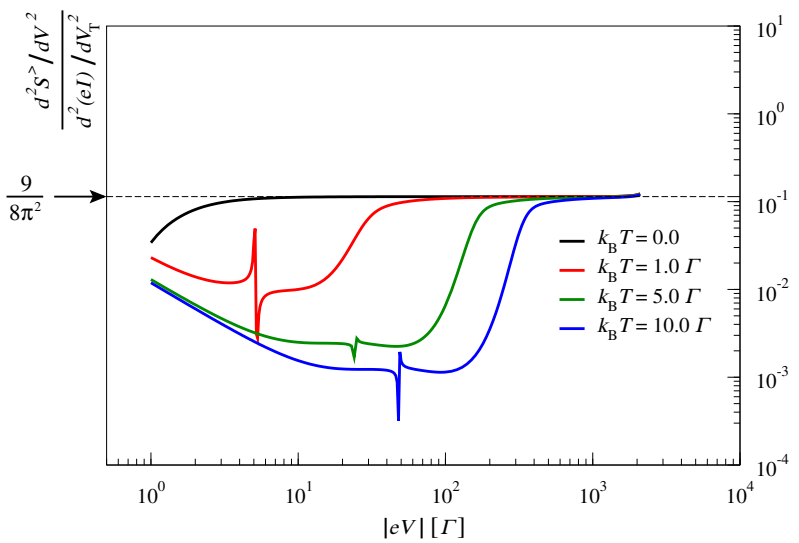

FIG. 9. The universal nonlinear response thermoelectric ratio of the second derivative of the greater currentcurrent correlator with respect to the bias voltage to the second derivative of the mean electric current times the electron charge with respect to the thermal voltage, $\left\{\partial^{2} S^{>}\left(V, V_{T}\right) / \partial V^{2}\right\} /\left\{\partial^{2}\left[e I\left(V, V_{T}\right)\right] / \partial V_{T}^{2}\right\}$. The thermal voltage is $e V_{T} / \Gamma=10^{-5}$. The Majorana overlap energy and the strength of the Majorana tunneling are $\xi / \Gamma=10^{-4}$ and $|\eta| / \Gamma=10^{3}$, respectively. The curves are shown for different temperatures and demonstrate that at $|e V| \gg k_{\mathrm{B}} T$ this universal nonlinear response thermoelectric ratio is remarkably robust against thermal fluctuations whose amplitude grows when the temperature increases.

however, we were unable to identify the universal asymptotic limit of the fluctuation thermoelectric coefficient $\partial S^{>}\left(V, V_{T}\right) / \partial V_{T}$. This high-energy universal law is definitely not a power dependence and it will be a challenge for future research.

In Fig. 7 we show the high-energy universal behavior of the fluctuation thermoelectric coefficient representing the second mixed derivative of the greater noise with respect to the thermal voltage and the bias voltage, $\partial^{2} S^{>}\left(V, V_{T}\right) / \partial V \partial V_{T}$, as a function of the bias voltage at $e V_{T} \ll \Gamma$ and for different temperatures. As one can see, at $|e V| \gg k_{\mathrm{B}} T$ the high-energy behavior of the fluctuation thermoelectric coefficient $\partial^{2} S^{>}\left(V, V_{T}\right) / \partial V \partial V_{T}$ is almost independent of the temperature and obeys the universal asymptotic limit,

$$
\frac{\partial^{2} S^{>}\left(V, V_{T}\right)}{\partial V \partial V_{T}}=-\frac{e^{4}}{\Gamma h} \ln (2)\left(\frac{|e V|}{\Gamma}\right)^{-3},
$$

shown in Fig. 7 as the dashed line. The high-energy universal behavior (36) follows from the high-energy universal behavior (34) of the fluctuation thermoelectric coefficient $\partial S^{>}\left(V, V_{T}\right) / \partial V_{T}$.

Comparison of the high-energy universal behavior of the mean thermoelectric coefficient $\partial^{2} I\left(V, V_{T}\right) / \partial V_{T}^{2}$ in Eq. 24 with the high-energy universal behavior of the fluctuation thermoelectric coefficient $\partial^{2} S^{>}\left(V, V_{T}\right) / \partial V \partial V_{T}$ in Eq. (36) shows that the ratio between these two nonlinear response coefficients is a truly 
universal constant with the asymptotic limit,

$$
\frac{\frac{\partial^{2} S^{>}\left(V, V_{T}\right)}{\partial V \partial V_{T}}}{\frac{\partial^{2}\left[e I\left(V, V_{T}\right)\right]}{\partial V_{T}^{2}}}=\frac{3 \ln (2)}{4 \pi^{2}},
$$

shown in the inset in Fig. 7 as the dashed horizontal line. As can be seen in the inset, indeed, at zero temperature and large bias voltages, $|e V| \gg \Gamma$, in the regime specified in 19 the thermoelectric ratio between $\partial^{2} S^{>}\left(V, V_{T}\right) / \partial V \partial V_{T}$ and $\partial^{2}\left[e I\left(V, V_{T}\right)\right] / \partial V_{T}^{2}$ is independent of $\epsilon_{d}, \Gamma, \eta$ and the bias voltage $V$. However, outside the high-energy regime, e.g., $|e V| \sim \Gamma$, or when the Majorana tunneling condition (19) is not satisfied, e.g., $|e V|>|\eta|$, one can see deviations from the truly universal value (37). The inset in Fig. 7 demonstrates that at low bias voltages there are strong deviations from the truly universal value (37). Since these deviations depend on $\Gamma$, the behavior at low bias voltages is not truly universal. Nevertheless the thermoelectric ratio between $\partial^{2} S^{>}\left(V, V_{T}\right) / \partial V \partial V_{T}$ and $\partial^{2}\left[e I\left(V, V_{T}\right)\right] / \partial V_{T}^{2}$ is still universal even at low bias voltages because it does not depend on $\epsilon_{d}$ in this voltage range. As expected, we find that outside the validity of the Majorana tunneling condition (19), e.g., $|e V|>|\eta|$, the thermoelectric ratio between $\partial^{2} S^{>}\left(V, V_{T}\right) / \partial V \partial V_{T}$ and $\partial^{2}\left[e I\left(V, V_{T}\right)\right] / \partial V_{T}^{2}$ is not universal (i.e. it depends on $\epsilon_{d}$ ) because the Majorana tunneling is not effective at such large bias voltages where the physics is not governed anymore by the Majorana fermions of the topological superconductor.

In Fig. 8 we show the high-energy universal behavior of the fluctuation nonlinear response coefficient representing the second derivative of the greater noise with respect to the bias voltage, $\partial^{2} S^{>}\left(V, V_{T}\right) / \partial V^{2}$, as a function of the bias voltage at $e V_{T} \ll \Gamma$ and for different temperatures. In the regime specified in $(23)$ the fluctuation nonlinear response coefficient $\partial^{2} S^{>}\left(V, V_{T}\right) / \partial V^{2}$ is pretty much insensitive at $|e V| \gg k_{\mathrm{B}} T$ to the temperature increase and its universal asymptotic limit is

$$
\frac{\partial^{2} S^{>}\left(V, V_{T}\right)}{\partial V^{2}}=-\frac{e^{4}}{\Gamma h} \frac{3}{2}\left(\frac{|e V|}{\Gamma}\right)^{-3} .
$$

Comparing Eq. 24) with Eq. (38) one obtains that the thermoelectric ratio between $\partial^{2} S^{>}\left(V, V_{T}\right) / \partial V^{2}$ and $\partial^{2}\left[e I\left(V, V_{T}\right)\right] / \partial V_{T}^{2}$ is a truly universal constant with the asymptotic limit

$$
\frac{\frac{\partial^{2} S^{>}\left(V, V_{T}\right)}{\partial V^{2}}}{\frac{\partial^{2}\left[e I\left(V, V_{T}\right)\right]}{\partial V_{T}^{2}}}=\frac{9}{8 \pi^{2}}
$$

in the regime specified in (23). This truly universal constant is shown in Fig. 9 as the dashed horizontal line. As demonstrated in Fig. 9, in the regime specified in (23) the universal Majorana thermoelectric ratio between the fluctuation nonlinear response coefficient $\partial^{2} S^{>}\left(V, V_{T}\right) / \partial V^{2}$ and the mean nonlinear coefficient $\partial^{2}\left[e I\left(V, V_{T}\right)\right] / \partial V_{T}^{2}$ is remarkably robust at $|e V| \gg k_{\mathrm{B}} T$ with respect to thermal noise excited at high temperatures.
At this point it is important to notice that the truly universal ratios 37 and 39 , which are valid in the Majorana tunneling regime (19) and at high bias voltages $|e V| \gg \Gamma$, might result from symmetries of the full counting statistics as has been discussed in Refs. 53 56. In particular, in Refs. [55, 56] symmetries of the full counting statistics were used to derive relations between nonlinear response coefficients beyond the Onsager-Casimir relation. Generalization of those relations between nonlinear response coefficients and derivation of (37) and (39) using symmetries of the full counting statistics in the presence of topological superconductors supporting Majorana bound states is an important fundamental task which is, to our knowledge, still unexplored and definitely represents a special topic for future research.

Let us estimate at which temperatures one can experimentally observe the truly universal high-energy Majorana plateau (39) in the thermoelectric ratio between the fluctuation nonlinear response coefficient $\partial^{2} S^{>}\left(V, V_{T}\right) / \partial V^{2}$ and the mean nonlinear response coefficient $\partial^{2} I\left(V, V_{T}\right) / \partial V_{T}^{2}$. We take the highest temperature shown in Fig. 9, that is $k_{\mathrm{B}} T=10 \Gamma$. In units of $|\eta|$ we have $k_{\mathrm{B}} T=0.01|\eta|$. Since $|\eta|$ is the largest energy scale, it should not exceed the induced superconducting energy gap $\Delta$ so as not to excite the bulk quasiparticles. Therefore, we assume $|\eta| \sim \Delta$. In Ref. 20] the induced superconducting energy gap is estimated as $\Delta \approx 250 \mu \mathrm{eV}$. One then obtains the temperature $T \approx 2.5 \mu \mathrm{eV} / k_{\mathrm{B}} \approx 0.03 \mathrm{~K}=30 \mathrm{mK}$. In Ref. [57] a higher value of $\Delta$ is reported, $\Delta \approx 15 \mathrm{meV}$. In this case one has $T \approx 1.8 \mathrm{~K}$. Such temperatures are already high enough and thus may easily be achieved in modern laboratories. As a consequence, the truly universal thermoelectric constant 39 represents a unique and highly conclusive Majorana signature to be detected in a realistic experiment.

\section{CONCLUSION}

In this work we have explored thermoelectric Majorana response of both the mean value and the fluctuations of the electric current. The research has been focused on unique universal Majorana thermoelectric signatures which may be detected in nanoscopic systems such as quantum dots. It has been shown that mean thermoelectric quantities become universal only at bias voltages high enough to exceed the overlap energy of the two Majorana bound states. For bias voltages below the Majorana overlap energy mean thermoelectric quantities are not universal even if the Majorana fermions are well separated. As a consequence, in order to obtain universal Majorana signatures from measurements of mean thermoelectric quantities one has to resort to essentially nonlinear response. In contrast, the thermoelectric response of the fluctuations of the electric current turns out to be universal at any bias voltage if the Majorana fermions are well separated. We have obtained various fluctuation thermoelectric coefficients in differ- 
ent transport regimes. In particular, it has been shown that the differential thermoelectric noise has a universal two plateau structure with the truly universal values of the plateaus $\left(e^{3} / h\right)[1+\ln (2)]$ and $\left(e^{3} / h\right)\left[1+\ln \left(2^{1 / 2}\right)\right]$ depending on whether the ratio between the bias voltage and the thermal voltage (characterizing the temperature difference between the contacts) is less than or greater than one. Further, universal high-energy behavior of the fluctuation nonlinear response coefficients has been presented and universal ratios between nonlinear response coefficients of the thermoelectric noise and the mean current have been obtained. Finally, we have demonstrated that at large bias voltages these thermoelectric ratios saturate to truly universal constants independent of the bias voltage and found the asymptotic limits of these truly universal constants. Importantly, these truly universal constants are protected by high bias voltages, making them robust against thermal noise. This robustness is crucial for realistic measurements. Therefore, the truly universal constants characterizing thermoelectric ratios at high bias voltages represent unique truly universal Majorana signatures challenging modern experiments on thermoelectric noise in quantum dots.

It is fair to mention that many issues within Majorana noise still remain to be addressed. In particular, the above results have been demonstrated for a simple model where only one single-particle energy level of the quantum dot is involved in transport. However, in many re- alistic systems several single-particle energy levels of the quantum dot may often contribute to transport. It is, therefore, interesting whether the results demonstrated here will change and, if so, what kind of Majorana fluctuation fingerprints one might expect in a multilevel system. This question as well as many other interesting issues will be a challenge for our future research on Majorana noise.

\section{ACKNOWLEDGMENTS}

The author thanks Milena Grifoni, Wataru Izumida and Meydi Ferrier for important discussions.

\section{Appendix: Basic steps in the calculation of the current-current correlator}

Taking the second derivative in Eq. 17 one easily finds that the current-current correlator is the sum of a one-particle, $S_{1_{l l^{\prime}}}\left(t, t^{\prime}\right)$, and a two-particle, $S_{2_{l l^{\prime}}}\left(t, t^{\prime}\right)$, terms,

$$
\left\langle I_{l}(t) I_{l^{\prime}}\left(t^{\prime}\right)\right\rangle=S_{1_{l l^{\prime}}}\left(t, t^{\prime}\right)+S_{2_{l l^{\prime}}}\left(t, t^{\prime}\right)
$$

The one-particle contribution has the form:

$$
\begin{aligned}
& S_{1_{l l^{\prime}}}\left(t, t^{\prime}\right)=\delta_{l l^{\prime}}\left|T_{l}\right|^{2}(-\mathrm{i})\left(\frac{e}{2 \hbar}\right)^{2} \sum_{k}\left\{\langle [ \psi _ { 1 } ( t ^ { \prime } ) + \psi _ { 2 } ( t ^ { \prime } ) ] [ - \overline { \psi } _ { 1 } ( t ) + \overline { \psi } _ { 2 } ( t ) ] \rangle _ { S _ { K } } \left[G_{l k}^{R}\left(t-t^{\prime}\right)+G_{l k}^{K}\left(t-t^{\prime}\right)-\right.\right. \\
& \left.\left.-G_{l k}^{A}\left(t-t^{\prime}\right)\right]+\left\langle\left[\psi_{1}(t)-\psi_{2}(t)\right]\left[\bar{\psi}_{1}\left(t^{\prime}\right)+\bar{\psi}_{2}\left(t^{\prime}\right)\right]\right\rangle_{S_{K}}\left[-G_{l k}^{R}\left(t^{\prime}-t\right)+G_{l k}^{K}\left(t^{\prime}-t\right)+G_{l k}^{A}\left(t^{\prime}-t\right)\right]\right\}
\end{aligned}
$$

where the angular brackets are defined in Eq. (15), $G_{l k}^{R, A, K}\left(t-t^{\prime}\right)$ are, respectively, the retarded, advanced and Keldysh Green's functions of the isolated contacts and we have performed the Keldysh rotation of the Grassmann fields of the quantum dot,

$$
\psi_{q}(t)=\frac{1}{\sqrt{2}}\left[\psi_{1}(t)+q \psi_{2}(t)\right], \quad \bar{\psi}_{q}(t)=\frac{1}{\sqrt{2}}\left[\bar{\psi}_{2}(t)+q \bar{\psi}_{1}(t)\right]
$$

The one-particle contribution in Eq. A.2 involves averages of products of only two Grassmann fields of the quantum dot. This contribution contains only normal terms, that is terms of the form $\left\langle\psi_{s}(t) \bar{\psi}_{s^{\prime}}\left(t^{\prime}\right)\right\rangle_{S_{K}}$, where $s, s^{\prime}=1,2$. It is obvious that anomalous terms, that is terms of the form $\left\langle\psi_{s}(t) \psi_{s^{\prime}}\left(t^{\prime}\right)\right\rangle_{S_{K}}$ or $\left\langle\bar{\psi}_{s}(t) \bar{\psi}_{s^{\prime}}\left(t^{\prime}\right)\right\rangle_{S_{K}}$, cannot appear in this case. However, such terms do arise when one averages products of four Grassmann fields in the two-particle contribution $S_{2_{l l^{\prime}}}\left(t, t^{\prime}\right)$.

The two-particle contribution has the form: 


$$
\begin{aligned}
& S_{2_{l l^{\prime}}}\left(t, t^{\prime}\right)=\left|T_{l}\right|^{2}\left|T_{l^{\prime}}\right|^{2} \frac{(-1)}{\hbar^{2}}\left(\frac{e}{2 \hbar}\right)^{2} \int_{-\infty}^{\infty} d t_{1} \int_{-\infty}^{\infty} d t_{2} \sum_{k_{1}, k_{2}}\left\langle\left\{[ - \psi _ { 1 } ( t ) + \psi _ { 2 } ( t ) ] \left[\bar{\psi}_{1}\left(t_{2}\right) G_{l k_{2}}^{R}\left(t_{2}-t\right)-\right.\right.\right. \\
& \left.-\bar{\psi}_{1}\left(t_{2}\right) G_{l k_{2}}^{K}\left(t_{2}-t\right)-\bar{\psi}_{2}\left(t_{2}\right) G_{l k_{2}}^{A}\left(t_{2}-t\right)\right]-\left[G_{l k_{2}}^{R}\left(t-t_{2}\right) \psi_{1}\left(t_{2}\right)+G_{l k_{2}}^{K}\left(t-t_{2}\right) \psi_{2}\left(t_{2}\right)-\right. \\
& \left.\left.-G_{l k_{2}}^{A}\left(t-t_{2}\right) \psi_{2}\left(t_{2}\right)\right]\left[-\bar{\psi}_{1}(t)+\bar{\psi}_{2}(t)\right]\right\}\left\{[ \psi _ { 1 } ( t ^ { \prime } ) + \psi _ { 2 } ( t ^ { \prime } ) ] \left[\bar{\psi}_{1}\left(t_{1}\right) G_{l^{\prime} k_{1}}^{R}\left(t_{1}-t^{\prime}\right)+\bar{\psi}_{1}\left(t_{1}\right) G_{l^{\prime} k_{1}}^{K}\left(t_{1}-t^{\prime}\right)+\right.\right. \\
& \left.+\bar{\psi}_{2}\left(t_{1}\right) G_{l^{\prime} k_{1}}^{A}\left(t_{1}-t^{\prime}\right)\right]-\left[G_{l^{\prime} k_{1}}^{R}\left(t^{\prime}-t_{1}\right) \psi_{1}\left(t_{1}\right)+G_{l^{\prime} k_{1}}^{K}\left(t^{\prime}-t_{1}\right) \psi_{2}\left(t_{1}\right)+\right. \\
& \left.\left.\left.+G_{l^{\prime} k_{1}}^{A}\left(t^{\prime}-t_{1}\right) \psi_{2}\left(t_{1}\right)\right]\left[\bar{\psi}_{1}\left(t^{\prime}\right)+\bar{\psi}_{2}\left(t^{\prime}\right)\right]\right\}\right\rangle_{S_{K}} .
\end{aligned}
$$

Since the total Keldysh action in Eq. (16) is quadratic, the average of four Grassmann fields is given as the sum of products of the averages of two Grassmann fields,

$$
\begin{aligned}
& \left\langle\psi_{1} \bar{\psi}_{2} \psi_{3} \bar{\psi}_{4}\right\rangle_{S_{K}}=\left\langle\psi_{1} \bar{\psi}_{2}\right\rangle_{S_{K}}\left\langle\psi_{3} \bar{\psi}_{4}\right\rangle_{S_{K}}- \\
& -\left\langle\psi_{1} \bar{\psi}_{4}\right\rangle_{S_{K}}\left\langle\psi_{3} \bar{\psi}_{2}\right\rangle_{S_{K}}-\left\langle\psi_{1} \psi_{3}\right\rangle_{S_{K}}\left\langle\bar{\psi}_{2} \bar{\psi}_{4}\right\rangle_{S_{K}}
\end{aligned}
$$

In Eq. A.5 $\psi_{1}, \bar{\psi}_{2}, \psi_{3}, \bar{\psi}_{4}$ schematically denote those Grassmann fields which are taken from a given product of four square brackets in Eq. A.4 in the same order as these Grassmann fields appear in these four square brackets.

All those terms in Eq. A.4 which correspond to the first term in the right hand side of Eq. A.5 do not represent interest for the calculation of the greater currentcurrent correlator $S^{>}\left(t, t^{\prime} ; V, V_{T}\right)$ in Eq. (27) because they give just the square of the mean electric current which is subtracted from the current-current correlator $\left\langle I_{L}(t) I_{L}\left(t^{\prime}\right)\right\rangle$ in Eq. 27).

All those terms in Eq. A.4 which correspond to the second and third terms in the right hand side of Eq. A.5 give, respectively, the normal and anomalous two-particle contributions to $S^{>}\left(t, t^{\prime} ; V, V_{T}\right)$.

To treat the normal and anomalous contributions to $S^{>}\left(t, t^{\prime} ; V, V_{T}\right)$ from both $S_{1_{l l^{\prime}}}\left(t, t^{\prime}\right)$ and $S_{2_{l l^{\prime}}}\left(t, t^{\prime}\right)$ it is convenient to introduce a particle-hole space via the Grassmann fields $\psi_{i s}(i=p, h ; s=1,2)$,

$$
\begin{array}{ll}
\psi_{i s}(t) \equiv \bar{\psi}_{s}(t), & i=p \\
\psi_{i s}(t) \equiv \psi_{s}(t), & i=h .
\end{array}
$$

The averages in Eqs. A.2 and A.4 are then expressed in terms of the hole-particle, hole-hole and particle-particle retarded, advanced and Keldysh Green's functions of the quantum dot via, respectively, the following relations:

$$
\begin{aligned}
\left\langle\psi_{h s}(t) \psi_{p s^{\prime}}\left(t^{\prime}\right)\right\rangle_{S_{K}} & =\left(\begin{array}{cc}
\mathrm{i} G_{h p}^{R}\left(t-t^{\prime}\right) & \mathrm{i} G_{h p}^{K}\left(t-t^{\prime}\right) \\
0 & \mathrm{i} G_{h p}^{A}\left(t-t^{\prime}\right)
\end{array}\right), \\
\left\langle\psi_{h s}(t) \psi_{h s^{\prime}}\left(t^{\prime}\right)\right\rangle_{S_{K}}= & =\left(\begin{array}{cc}
\mathrm{i} G_{h h}^{K}\left(t-t^{\prime}\right) & \mathrm{i} G_{h h}^{R}\left(t-t^{\prime}\right) \\
\mathrm{i} G_{h h}^{A}\left(t-t^{\prime}\right) & 0
\end{array}\right), \\
\left\langle\psi_{p s}(t) \psi_{p s^{\prime}}\left(t^{\prime}\right)\right\rangle_{S_{K}} & =\left(\begin{array}{cc}
0 & \mathrm{i} G_{p p}^{A}\left(t-t^{\prime}\right) \\
\mathrm{i} G_{p p}^{R}\left(t-t^{\prime}\right) & \mathrm{i} G_{p p}^{K}\left(t-t^{\prime}\right)
\end{array}\right) .
\end{aligned}
$$

Since the total Keldysh action in Eq. (16) is quadratic, the calculation of the Green's functions in the right hand sides of Eqs. A.7)- A.9 is reduced to calculations of the corresponding elements of the inverse kernel of this action. This is a quite simple, although somewhat lengthy, mathematical step which one may easily perform. After this step one finds the expressions for the hole-particle, hole-hole and particle-particle retarded, advanced and Keldysh Green's functions of the quantum dot. All the retarded and advanced Green's functions have already been found in Ref. [36] in the energy domain. For completeness we give them here:

$$
\begin{aligned}
& G_{h p}^{R}(\epsilon)=\frac{N_{h p}^{R}(\epsilon)}{f(\epsilon)}, \quad G_{h h}^{R}(\epsilon)=\frac{-8 \hbar\left(\eta^{*}\right)^{2} \epsilon}{f(\epsilon)}, \quad G_{p p}^{R}(\epsilon)=\frac{-8 \hbar \eta^{2} \epsilon}{f(\epsilon)}, \\
& G_{h p}^{A}(\epsilon)=\left[G_{h p}^{R}(\epsilon)\right]^{*}, \quad G_{h h}^{A}(\epsilon)=\left[G_{p p}^{R}(\epsilon)\right]^{*}, \quad G_{p p}^{A}(\epsilon)=\left[G_{h h}^{R}(\epsilon)\right]^{*}, \\
& f(\epsilon)=4 \epsilon^{4}-\epsilon^{2}\left(\Gamma^{2}+4 \epsilon_{d}^{2}+4 \xi^{2}+16|\eta|^{2}\right)+\xi^{2}\left(\Gamma^{2}+4 \epsilon_{d}^{2}\right)+\mathrm{i} 4 \Gamma\left[\epsilon^{3}-\epsilon\left(\xi^{2}+2|\eta|^{2}\right)\right], \\
& N_{h p}^{R}(\epsilon)=2 \hbar\left\{-4|\eta|^{2} \epsilon-\left(\xi^{2}-\epsilon^{2}\right)\left[\mathrm{i} \Gamma+2\left(\epsilon_{d}+\epsilon\right)\right]\right\} .
\end{aligned}
$$


Finally, for the Keldysh components of the hole-particle, hole-hole and particle-particle Green's functions one obtains the following expressions:

$$
\begin{aligned}
& G_{h p}^{K}(\epsilon)=\frac{N_{h p}^{K}(\epsilon)}{|f(\epsilon)|^{2}}, \quad G_{h h}^{K}(\epsilon)=\frac{N_{h h}^{K}(\epsilon)}{|f(\epsilon)|^{2}}, \quad G_{p p}^{K}(\epsilon)=\frac{N_{p p}^{K}(\epsilon)}{|f(\epsilon)|^{2}}, \\
& N_{h p}^{K}(\epsilon)=-2 \mathrm{i} \Gamma \hbar\left\{\left(F_{L}(\epsilon)+F_{R}(\epsilon)\right)\left(\xi^{2}-\epsilon^{2}\right)^{2}\left[\Gamma^{2}+4\left(\epsilon_{d}+\epsilon\right)^{2}\right]+\right. \\
& \left.+16|\eta|^{2} \epsilon\left[\left(F_{L}(\epsilon)+F_{R}(\epsilon)\right)\left(\xi^{2}-\epsilon^{2}\right)\left(\epsilon_{d}+\epsilon\right)+|\eta|^{2} \epsilon\left(F_{L}(\epsilon)-F_{L}(-\epsilon)+F_{R}(\epsilon)-F_{R}(-\epsilon)\right)\right]\right\}, \\
& N_{h h}^{K}(\epsilon)=8 \Gamma \hbar\left(\eta^{*}\right)^{2} \epsilon\left\{( \xi ^ { 2 } - \epsilon ^ { 2 } ) \left[\left(F_{L}(\epsilon)+F_{L}(-\epsilon)+F_{R}(\epsilon)+F_{R}(-\epsilon)\right)\left(\Gamma-2 \mathrm{i} \epsilon_{d}\right)+\right.\right. \\
& \left.\left.+2 \mathrm{i} \epsilon\left(F_{L}(-\epsilon)-F_{L}(\epsilon)+F_{R}(-\epsilon)-F_{R}(\epsilon)\right)\right]+4 \mathrm{i}|\eta|^{2} \epsilon\left(F_{L}(-\epsilon)-F_{L}(\epsilon)+F_{R}(-\epsilon)-F_{R}(\epsilon)\right)\right\}, \\
& N_{p p}^{K}(\epsilon)=-\left(N_{h h}^{K}(\epsilon)\right)^{*},
\end{aligned}
$$

where $F_{L, R}(\epsilon) \equiv 1-2 f_{L, R}(\epsilon)$.

Using Eqs. A.2), (A.4), A.5 and (A.7)- A.11) one may without much effort express the zero frequency noise in Eq. (29) as well as its various derivatives as integrals in the energy domain. These integrals may be calculated numerically to obtain the quantities discussed in the main text as functions of the bias voltage and thermal voltage.
[1] E. Majorana, "Teoria simmetrica dell'elettrone e del positrone," Nuovo Cimento 14, 171 (1937).

[2] C. Itzykson and J.-B. Zuber, Quantum Field Theory (McGraw-Hill, New York, St. Louis, San Francisco, 1980).

[3] A. Yu. Kitaev, "Unpaired Majorana fermions in quantum wires," Phys.-Usp. 44, 131 (2001).

[4] J. Alicea, "New directions in the pursuit of Majorana fermions in solid state systems," Rep. Prog. Phys. 75, 076501 (2012).

[5] M. Leijnse and K. Flensberg, "Introduction to topological superconductivity and Majorana fermions," Semicond. Sci. Technol. 27, 124003 (2012).

[6] M. Sato and S. Fujimoto, "Majorana fermions and topology in superconductors," J. Phys. Soc. Japan 85, 072001 (2016).

[7] L. Fu and C. L. Kane, "Superconducting proximity effect and Majorana fermions at the surface of a topological insulator," Phys. Rev. Lett. 100, 096407 (2008).

[8] L. Fu and C. L. Kane, "Josephson current and noise at a superconductor/quantum-spin-Hallinsulator/superconductor junction," Phys. Rev. B 79, 161408(R) (2009).

[9] R. M. Lutchyn, J. D. Sau, and S. Das Sarma, "Majorana fermions and a topological phase transition in semiconductor-superconductor heterostructures," Phys. Rev. Lett. 105, 077001 (2010).

[10] Y. Oreg, G. Refael, and F. von Oppen, "Helical liquids and Majorana bound states in quantum wires," Phys. Rev. Lett. 105, 177002 (2010).

[11] L. Fidkowski, J. Alicea, N. H. Lindner, R. M. Lutchyn, and M. P. A. Fisher, "Universal transport signatures of Majorana fermions in superconductor-Luttinger liquid junctions," Phys. Rev. B 85, 245121 (2012).

[12] M. Lee, J. S. Lim, and R. López, "Kondo effect in a quantum dot side-coupled to a topological superconductor," Phys. Rev. B 87, 241402(R) (2013).

[13] A. Kundu and B. Seradjeh, "Transport signatures of Flo- quet Majorana fermions in driven topological superconductors," Phys. Rev. Lett. 111, 136402 (2013).

[14] R. Ilan, J. H. Bardarson, H.-S. Sim, and J. E. Moore, "Detecting perfect transmission in Josephson junctions on the surface of three dimensional topological insulators," New J. Phys. 16, 053007 (2014).

[15] M. Cheng, M. Becker, B. Bauer, and R. M. Lutchyn, "Interplay between Kondo and Majorana interactions in quantum dots," Phys. Rev. X 4, 031051 (2014).

[16] A. M. Lobos and S. Das Sarma, "Tunneling transport in NSN Majorana junctions across the topological quantum phase transition," New J. Phys. 17, 065010 (2015).

[17] R. Wang, H.-Y. Lu, B. Wang, and C. S. Ting, "Spinon Majorana fermions," Phys. Rev. B 94, 125146 (2016).

[18] R. M. Lutchyn and L. I. Glazman, "Transport through a Majorana island in the strong tunneling regime," Phys. Rev. Lett. 119, 057002 (2017).

[19] H. Huang, Q.-F. Liang, D.-X. Yao, and Z. Wang, "Majorana $\phi_{0}$-junction in a disordered spin-orbit coupling nanowire with tilted magnetic field," Physica C: Superconductivity and its Applications 543, 22 (2017).

[20] V. Mourik, K. Zuo, S. M. Frolov, S. R. Plissard, E. P. A. M. Bakkers, and L. P. Kouwenhoven, "Signatures of Majorana fermions in hybrid superconductorsemiconductor nanowire devices," Science 336, 1003 (2012).

[21] S. M. Albrecht, A. P. Higginbotham, M. Madsen, F. Kuemmeth, T. S. Jespersen, J. Nygård, P. Krogstrup, and C. M. Marcus, "Exponential protection of zero modes in Majorana islands," Nature 531, 206 (2016).

[22] H. Zhang, C.-X. Liu, S. Gazibegovic, D. Xu, J. A. Logan, G. Wang, N. van Loo, J. D. S. Bommer, M. W. A. de Moor, D. Car, R. L. M. O. het Veld, P. J. van Veldhoven, S. Koelling, M. A. Verheijen, M. Pendharkar, D. J. Pennachio, B. Shojaei, J. S. Lee, C. J. Palmstrøm, E. P. A. M. Bakkers, S. D. Sarma, and L. P. Kouwenhoven, "Quantized Majorana conductance," Nature 556, 74 (2018). 
[23] G. Kells, D. Meidan, and P. W. Brouwer, "Near-zeroenergy end states in topologically trivial spin-orbit coupled superconducting nanowires with a smooth confinement," Phys. Rev. B 86, 100503(R) (2012).

[24] C.-X. Liu, J. D. Sau, T. D. Stanescu, and S. D. Sarma, "Andreev bound states versus Majorana bound states in quantum dot-nanowire-superconductor hybrid structures: Trivial versus topological zero-bias conductance peaks," Phys. Rev. B 96, 075161 (2017).

[25] E. Prada, R. Aguado, and P. San-Jose, "Measuring Majorana nonlocality and spin structure with a quantum dot," Phys. Rev. B 96, 085418 (2017).

[26] D. J. Clarke, "Experimentally accessible topological quality factor for wires with zero energy modes," Phys. Rev. B 96, 201109(R) (2017).

[27] D. E. Liu, M. Cheng, and R. M. Lutchyn, "Probing Majorana physics in quantum-dot shot-noise experiments," Phys. Rev. B 91, 081405(R) (2015).

[28] D. E. Liu, A. Levchenko, and R. M. Lutchyn, "Majorana zero modes choose Euler numbers as revealed by full counting statistics," Phys. Rev. B 92, 205422 (2015).

[29] C. W. J. Beenakker, "Random-matrix theory of Majorana fermions and topological superconductors," Rev. Mod. Phys. 87, 1037 (2015).

[30] S. Valentini, M. Governale, R. Fazio, and F. Taddei, "Finite-frequency noise in a topological superconducting wire," Physica E 75, 15-21 (2016).

[31] H. Nyquist, "Thermal agitation of electric charge in conductors," Phys. Rev. 32, 110 (1928).

[32] H. B. Callen and T. A. Welton, "Irreversibility and generalized noise," Phys. Rev. 83, 34 (1951).

[33] L. D. Landau and E. M. Lifshitz, Statistical Physics. Part 1: Course of Theoretical Physics, 3rd ed., Vol. 5 (Pergamon Press, Oxford, 1980).

[34] A. Haim, E. Berg, F. von Oppen, and Y. Oreg, "Current correlations in a Majorana beam splitter," Phys. Rev. B 92, 245112 (2015).

[35] S. Smirnov, "Non-equilibrium Majorana fluctuations," New J. Phys. 19, 063020 (2017).

[36] S. Smirnov, "Majorana tunneling entropy," Phys. Rev. B 92, 195312 (2015).

[37] M. Ferrier, T. Arakawa, T. Hata, R. Fujiwara, R. Delagrange, R. Weil, R. Deblock, R. Sakano, A. Oguri, and K. Kobayashi, "Universality of non-equilibrium fluctuations in strongly correlated quantum liquids," Nature Physics 12, 230 (2016).

[38] T. Senthil and M. P. A. Fisher, "Quasiparticle localization in superconductors with spin-orbit scattering," Phys. Rev. B 61, 9690 (2000).

[39] N. Read and D. Green, "Paired states of fermions in two dimensions with breaking of parity and time-reversal symmetries and the fractional quantum Hall effect," Phys. Rev. B 61, 10267 (2000).

[40] T. Mizushima and M. Nitta, "Topology and symmetry of surface Majorana arcs in cyclic superconductors," Phys. Rev. B 97, 024506 (2018).

[41] A. Dorda, M. Ganahl, S. Andergassen, W. von der Lin- den, and E. Arrigoni, "Thermoelectric response of a correlated impurity in the nonequilibrium Kondo regime," Phys. Rev. B 94, 245125 (2016).

[42] I. Titvinidze, A. Dorda, W. von der Linden, and E. Arrigoni, "Thermoelectric properties of a strongly correlated layer," Phys. Rev. B 96, 115104 (2017).

[43] M. Leijnse, "Thermoelectric signatures of a Majorana bound state coupled to a quantum dot," New J. Phys. 16, 015029 (2014).

[44] R. López, M. Lee, L. Serra, and J. S. Lim, "Thermoelectrical detection of Majorana states," Phys. Rev. B 89, 205418 (2014)

[45] H. Khim, R. López, J. S. Lim, and M. Lee, "Thermoelectric effect in the Kondo dot side-coupled to a Majorana mode," Eur. Phys. J. B 88, 151 (2015).

[46] J. P. Ramos-Andrade, O. Ávalos-Ovando, P. A. Orellana, and S. E. Ulloa, "Thermoelectric transport through Majorana bound states and violation of Wiedemann-Franz law," Phys. Rev. B 94, 155436 (2016).

[47] A. C. Hewson, The Kondo Problem to Heavy Fermions (Cambridge University Press, Cambridge, 1997).

[48] J. Fuchs and C. Schweigert, Symmetries, Lie Algebras and Representations, a Graduate Course for Physicists (Cambridge University Press, Cambridge, 1997).

[49] A. Kamenev and A. Andreev, "Electron-electron interactions in disordered metals: Keldysh formalism," Phys. Rev. B 60, 2218 (1999).

[50] A. Altland and B. Simons, Condensed Matter Field Theory, 2nd ed. (Cambridge University Press, Cambridge, 2010).

[51] Y. Meir and N. S. Wingreen, "Landauer formula for the current through an interacting electron region," Phys. Rev. Lett. 68, 2512 (1992).

[52] D. Goldhaber-Gordon, H. Shtrikman, D. Mahalu, D. Abusch-Magder, U. Meirav, and M. A. Kastner, "Kondo effect in a single-electron transistor," Nature 391, 156 (1998).

[53] J. Tobiska and Yu. V. Nazarov, "Inelastic interaction corrections and universal relations for full counting statistics in a quantum contact," Phys. Rev. B 72, 235328 (2005).

[54] D. Andrieux and P. Gaspard, "Fluctuation theorem for transport in mesoscopic systems," J. Stat. Mech. 2006, P01011 (2006).

[55] K. Saito and Y. Utsumi, "Symmetry in full counting statistics, fluctuation theorem, and relations among nonlinear transport coefficients in the presence of a magnetic field," Phys. Rev. B 78, 115429 (2008).

[56] E. Iyoda, Y. Utsumi, and T. Kato, "Nonequilibrium extension of Onsager relations for thermoelectric effects in mesoscopic conductors," J. Phys. Soc. Japan 79, 045003 (2010).

[57] E. Wang, H. Ding, A. V. Fedorov, W. Yao, Z. Li, Y.F. Lv, K. Zhao, L.-G. Zhang, Z. Xu, J. Schneeloch, R. Zhong, S.-H. Ji, L. Wang, K. He, X. Ma, G. Gu, H. Yao, Q.-K. Xue, X. Chen, and S. Zhou, "Fully gapped topological surface states in $\mathrm{Bi}_{2} \mathrm{Se}_{3}$ films induced by a $d$ wave high-temperature superconductor," Nature Physics 9, 621 (2013). 